\title{
\begin{tabular}{l|l} 
Mibraries & DSpace@MIT
\end{tabular}
}

\author{
MIT Open Access Articles
}

\section{Moist Formulations of the Eliassen-Palm Flux and Their Connection to the Surface Westerlies}

The MIT Faculty has made this article openly available. Please share how this access benefits you. Your story matters.

Citation: Dwyer, John G., and O'Gorman, Paul A. "Moist Formulations of the Eliassen-Palm Flux and Their Connection to the Surface Westerlies." Journal of the Atmospheric Sciences 74, 2 (February 2017): 513-530 (c) 2017 American Meteorological Society

As Published: http://dx.doi.org/10.1175/jas-d-16-0111.1

Publisher: American Meteorological Society

Persistent URL: http://hdl.handle.net/1721.1/111124

Version: Final published version: final published article, as it appeared in a journal, conference proceedings, or other formally published context

Terms of Use: Article is made available in accordance with the publisher's policy and may be subject to US copyright law. Please refer to the publisher's site for terms of use. 


\title{
Moist Formulations of the Eliassen-Palm Flux and Their Connection to the Surface Westerlies
}

\author{
John G. Dwyer and Paul A. O'GORMan \\ Department of Earth, Atmospheric and Planetary Sciences, Massachusetts Institute of Technology, \\ Cambridge, Massachusetts
}

(Manuscript received 8 April 2016, in final form 31 October 2016)

\begin{abstract}
The Eliassen-Palm (EP) flux is an important diagnostic for wave propagation and wave-mean flow interaction in the atmosphere. Here, two moist formulations of the EP flux are compared with the traditional dry EP flux, and their links to the surface westerlies are analyzed using reanalysis data and simulations with GCMs. The first moist formulation of the EP flux modifies only the static stability to account for latent heat release by eddies, while the second moist formulation simply replaces all potential temperatures with equivalent potential temperatures. For reanalysis data, the peak upward EP flux in the lower troposphere is farther equatorward and stronger when the moist formulations are used, with greater changes for the second moist formulation. The moist formulations have the advantage of giving a closer relationship over the seasonal cycle between the latitudes of the peak surface westerlies and the peak upward EP flux. In simulations with a comprehensive GCM, the dry and moist upward EP fluxes shift poleward by a similar amount as the climate warms. In simulations over a wider range of climates with an idealized GCM, the surface westerlies can shift both poleward and equatorward with warming, and they are influenced by an anomalous region of dry EP flux divergence near the subtropical jet. Using moist EP fluxes weakens this anomalous divergence in the idealized GCM simulations, and the shifts in the surface westerlies can then be understood through changes in the preference for equatorward or poleward wave propagation.
\end{abstract}

\section{Introduction}

Eliassen-Palm (EP) fluxes are often thought of as representing the effect of transient and stationary eddy fluxes on the zonal-mean circulation. But their standard definition only includes eddy fluxes of heat and momentum, even though condensational heating associated with eddy latent heat transport can change the mean temperature gradients and thus the zonal flow via the thermal wind relation. Explicitly including the effect of eddy latent heating in the EP fluxes could provide a more complete description of the influence of eddies on the zonal-mean flow. EP fluxes are also an important diagnostic for wave propagation, and from this perspective it is also desirable for them to account for moisture since latent heating is known to affect baroclinic wave evolution (e.g., Emanuel et al. 1987).

Eddy transport of moisture is important both at low latitudes, for example in monsoons and their associated

Corresponding author e-mail: John G. Dwyer, jgdwyer@mit.edu stationary subtropical anticyclones (Shaw and Pauluis 2012), and in the midlatitudes, where roughly half of the poleward energy transport is in the form of latent heat (Trenberth and Stepaniak 2003). And as Earth's climate warms, it becomes even more important to understand the role of moisture because of the strong dependence of specific humidity on temperature: around $7 \% \mathrm{~K}^{-1}$ for typical lower-tropospheric temperatures. A better understanding of the relationship between moisture and dynamics may be expected to give insights into the response to climate change, such as the poleward shift of the storm tracks and associated surface westerlies that is projected to occur by GCMs (Kushner et al. 2001; Yin 2005).

Previous studies have investigated the role of moisture for the momentum budget and for the meridional mass transport. Stone and Salustri (1984) modified the quasigeostrophic (QG) EP flux to account for the effect of latent heat release and found an approximate doubling of the eddy forcing on the zonal-mean zonal wind. Tung (1986) derived a nongeostrophic EP flux in dry 
isentropic coordinates that includes cross-isentropic momentum transport associated with diabatic heating. Recent studies emphasize that the meridional circulation is stronger on moist isentropes than dry isentropes (Pauluis et al. 2008) and in the moist statistical transformed Eulerian mean (TEM) as compared to the dry TEM (Pauluis et al. 2011). Because equivalent potential temperature is not a monotonic function of height, directly calculating EP fluxes on moist isentropes is challenging. Recent approaches have addressed this issue by using the mass above isentropes as a vertical coordinate (Chen 2013) or by using a weak formulation (involving a vertical integral) of the mass-weighted zonal average (Yamada and Pauluis 2016).

Our focus in this study is to explore the link between moisture and momentum balance in the midlatitudes. In particular, we are interested in using moist theories to understand the positioning of the surface westerlies in current and future climates. We approach this problem by defining moist EP fluxes in two different ways. The first modifies the static stability to account for latent heat release by eddies using the effective static stability of O'Gorman (2011), while the second approach replaces potential temperature in the thermodynamic equation with its moist analog, equivalent potential temperature, broadly following Stone and Salustri (1984). Both approaches include the effects of eddy latent heat release in a relatively straightforward way, and they have different advantages and drawbacks as discussed in detail in section 2. For example, the effective static stability gives an EP flux that can be linked to wave propagation and a wave activity, but it does not easily connect to the transformed Eulerian mean since it only applies to eddies, while replacing potential temperatures with equivalent potential temperatures allows for the formulation of a moist TEM, but it does not allow for a connection to a wave activity or a conserved potential vorticity (PV).

Previous work suggests that the latitude of the surface westerlies is linked to the latitude of the vertical component of the EP flux in the lower troposphere ( $\mathrm{Lu}$ et al. 2010; Donohoe et al. 2014). This is a useful property for building theories to explain shifts in the surface westerlies. Lu et al. (2010) used the dry EP flux to connect changes in the position of the surface westerlies to changes in the SST distribution in an aquaplanet GCM, and Donohoe et al. (2014) used the moist EP flux of Stone and Salustri (1984) to relate the surface westerlies to the meridional atmospheric eddy energy flux also in an aquaplanet GCM. Here we explore the link between surface westerlies and EP fluxes over the seasonal cycle and under climate change using reanalysis data, a comprehensive GCM, and an idealized GCM. The surface westerlies shift poleward with warming in the comprehensive GCM simulation, but they can shift either poleward or equatorward with warming over parts of the range of climates simulated by the idealized GCM (Schneider et al. 2010). This more complicated behavior of the surface westerlies, together with the wide range of simulated climates (including very hot and humid climates), make the idealized GCM simulations an interesting test case for the application of moist EP fluxes.

We also study whether the moist EP fluxes can improve our understanding of a longstanding, peculiar feature of observations: an anomalous region of dry EP flux divergence on the poleward flank of the subtropical jet in the upper troposphere and lower stratosphere (Edmon Jr. et al. 1980; Karoly 1982; Birner et al. 2013). The free atmosphere is mainly characterized by EP flux convergence, understood to be a consequence of downgradient eddy PV fluxes, but this anomalous region is associated with upgradient eddy PV fluxes. Birner et al. (2013) provided evidence that these upgradient or "antidiffusive" eddy PV fluxes are balanced in the potential enstrophy budget by the local convergence of poleward enstrophy fluxes primarily from planetary-scale waves. Note that the upgradient eddy PV fluxes have also been found in an analysis limited to transient eddies (Bartels et al. 1998). The analysis by Birner et al. (2013) did not explicitly include diabatic effects, but it has previously been suggested that diabatic effects could be a contributor to the anomalous EP flux divergence (Karoly 1982). We find here that the anomalous EP flux divergence is of similar strength in both the dry and moist EP flux formulations, which suggests that moist processes do not strongly contribute to it in both the reanalysis and the comprehensive GCM. But in the idealized GCM, the anomalous EP flux divergence is reduced in magnitude when moist EP fluxes are used, and we investigate it further by analyzing the eddy potential enstrophy budget.

The rest of this paper is laid out as follows. In section 2 we describe the moist formulations of the EP fluxes. Then in section 3 we compare the moist EP fluxes to the dry EP fluxes using reanalysis data and analyze their projected future changes using a comprehensive GCM. We also evaluate the relationship between EP fluxes and surface westerlies in the current and future climates. Next, in section 4 we study the relationships between the dry and moist EP fluxes and the surface westerlies over a wide range of climates in idealized GCM simulations, including the role of the anomalous region of EP flux divergence and changes in the preference for equatorward or poleward wave propagation. We then summarize our results and their implications in section 5 . 


\section{EP flux theory}

The (dry) EP flux in pressure coordinates on the sphere is given by $\mathbf{F}=\left(F^{(\phi)}, F^{(p)}\right)$, where

$$
\begin{aligned}
& F^{(\phi)}=a \cos \phi\left(-\overline{u^{\prime} v^{\prime}}+\frac{\overline{v^{\prime} \theta^{\prime}}}{\bar{\theta}_{p}} \bar{u}_{p}\right) \\
& F^{(p)}=a \cos \phi\left\{\frac{\bar{v}^{\prime} \theta^{\prime}}{\bar{\theta}_{p}}\left[f-\frac{(\bar{u} \cos \phi)_{\phi}}{a \cos \phi}\right]-\overline{u^{\prime} \omega^{\prime}}\right\},
\end{aligned}
$$

and $a$ is Earth's radius; $\phi$ is latitude; $u$ and $v$ are the zonal and meridional winds, respectively; $\theta$ is potential temperature; $f$ is the Coriolis parameter; $\omega$ is pressure velocity; subscripts denote partial derivatives; and eddies (primed quantities) are taken with respect to the zonal mean, as denoted with an overbar (e.g., Andrews et al. 1983). While we use this definition of the EP flux when calculating our results, it is also conceptually helpful to consider the EP flux in the QG approximation, given by

$$
\begin{aligned}
& F^{(\phi)}=-a \cos \phi \overline{u^{\prime} v^{\prime}} \\
& F^{(p)}=a \cos \phi f \frac{\overline{v^{\prime} \theta^{\prime}}}{\bar{\theta}_{p}} .
\end{aligned}
$$

The properties of EP fluxes have been discussed at length in previous work (e.g., Eliassen and Palm 1961; Andrews and McIntyre 1976, 1978; Edmon Jr. et al. 1980; Tung 1986), and we summarize them here in the QG limit for simplicity. Briefly, the EP flux is a useful diagnostic of the propagation of waves (both magnitude and direction) in the meridional plane. For smallamplitude waves, it represents the flux of wave activity, and its divergence is zero for steady conservative waves. The QG TEM momentum equation is

$$
\frac{\partial \bar{u}}{\partial t}=f \bar{v}^{\dagger}+\overline{\mathscr{F}}+\frac{1}{a \cos \phi} \nabla \cdot \mathbf{F},
$$

where $v^{\dagger}=v-\left(\overline{v^{\prime} \theta^{\prime}} / \bar{\theta}_{p}\right)_{p}$ is the residual-mean meridional velocity, $\nabla \cdot \mathbf{F}=(a \cos \phi)^{-1} \partial_{\phi}\left[F^{(\phi)} \cos \phi\right]+\partial_{p} F^{(p)}$, and $\overline{\mathscr{F}}$ is the zonal-mean frictional force. This equation shows that the divergence of the EP flux represents the combined forcing of the mean zonal wind by eddy fluxes of momentum and heat.

The divergence of the QG EP flux is also related to the QG eddy potential vorticity flux as

$$
\nabla \cdot \mathbf{F}=a \cos \phi \overline{v^{\prime} q^{\prime}},
$$

where $q^{\prime}=\zeta^{\prime}+f\left(\theta^{\prime} / \bar{\theta}_{p}\right)_{p}$, with $\zeta$ the relative vorticity. This local QG relationship assumes that the latitudinal dependence of the static stability and $f$ are weak: over the eddy length scale, $\Delta \bar{\theta}_{p} / \bar{\theta}_{p}$ and $\Delta f / f$ should be on the order of the Rossby number or smaller (Edmon Jr. et al. 1980). While often appropriate for the dry case away from the tropopause, this assumption becomes less accurate when a moist static stability is used because of meridional humidity gradients, and this is one of the reasons we use the full (non QG) EP flux in all of our results.

We will see below that neither of the moist EP fluxes satisfy all of the properties that dry EP fluxes do, and one may question whether "moist EP flux" is an appropriate term. We follow previous authors (Yamada and Pauluis 2016) in using the term "moist EP flux" because of the close links with the dry EP flux.

\section{a. Moist EP flux: Effective static stability}

The first moist EP flux formulation is defined by replacing the static stability $\bar{\theta}_{p}$ in Eq. (1) with the effective static stability of O'Gorman (2011). The effective static stability approximately accounts for both the moist static stability in regions of ascent (which are assumed to be saturated) and the dry static stability in regions of descent. The derivation of the effective static stability given in O'Gorman (2011) is only valid for the eddy thermodynamic equation; the mean thermodynamic equation still includes the dry static stability and a mean latent heating term. Latent heat release is assumed to occur where there is upward motion, and the resulting nonlinear dependence of latent heating on the vertical velocity is accounted for using an asymmetry parameter $\lambda$, which measures the up-down asymmetry of the vertical velocity. The parameter $\lambda$ is calculated as the regression of the eddy upward vertical velocity $\omega^{\uparrow \prime}$ (a proxy for the eddy latent heating rate, where $\omega^{\uparrow}=\omega$ when $\omega<0$, and $\omega^{\uparrow}=0$ otherwise) on the eddy vertical velocity $\omega^{\prime}$. The regression is performed using 6-hourly data at each latitude and level over all longitudes and times, and $\lambda$ is found to be roughly constant in the extratropical free troposphere with values of $\lambda \approx 0.6$, corresponding to faster upward than downward motion. The effective static stability at a given latitude and level is then defined as

$$
-\left(\frac{\partial \theta}{\partial p}\right)_{\mathrm{eff}}=-\frac{\partial \bar{\theta}}{\partial p}+\left.\lambda \frac{\partial \theta}{\partial p}\right|_{\theta^{*}},
$$

where $\theta^{*}$ is the saturated equivalent potential temperature, and $-\partial \theta /\left.\partial p\right|_{\theta^{*}}$ is the dry static stability for a moist adiabatic stratification at the mean temperature and pressure. At very low temperatures, the effective static stability reduces to the dry case since $\partial \theta /\left.\partial p\right|_{\theta^{*}}$ tends to zero. As the second term on the right-hand side of 
Eq. (5) is negative, the effective static stability is smaller than the dry static stability, reflecting the reduced stratification that eddies experience in a moist atmosphere.

We define an effective QG eddy $\mathrm{PV}$ as $q_{\mathrm{eff}}^{\prime}=\zeta^{\prime}+f\left[\theta^{\prime} /\left(\theta_{p}\right)_{\mathrm{eff}}\right]_{p}$, which leads to the same equivalence between the effective QG eddy PV flux and the effective EP flux divergence as given in Eq. (4) for the dry case. According to O'Gorman (2011), the eddy thermodynamic equation written using the effective static stability has the same form as in the dry case but without the latent heating term, and it may be combined with the eddy vorticity equation to give an evolution equation for the effective QG eddy PV. For the midlatitude beta plane and neglecting second-order terms, this equation is $\partial_{t} q_{\mathrm{eff}}^{\prime}=-\bar{u} \partial_{x} q_{\mathrm{eff}}^{\prime}-v^{\prime}\left(\partial_{y} q\right)_{\mathrm{eff}}+S^{\prime}$, where $\left(\partial_{y} q\right)_{\text {eff }}=\beta+f\left[\bar{\theta}_{y} /\left(\theta_{p}\right)_{\text {eff }}\right]_{p}$, with $\beta$ the meridional gradient of the Coriolis parameter, and where $S^{\prime}$ is the eddy tendency due to radiation and friction. The above equation for $\partial_{t} q_{\text {eff }}^{\prime}$ has the same form as the dry QG eddy $\mathrm{PV}$ equation and implies a conservation equation for effective wave activity (density) in the smallamplitude QG limit: $\partial_{t} A_{\text {eff }}+\nabla \cdot \mathbf{F}_{\text {eff }}=D$, where $A_{\text {eff }}=(1 / 2) \overline{q_{\text {eff }}^{\prime 2}} /\left(\partial_{y} q\right)_{\text {eff }}$ and $D$ represents the tendency due to radiation and friction. Similarly, the effective $Q G$ eddy potential enstrophy equation may be derived, and the relation of the EP flux to the group velocity and effective wave activity also follows (not shown).

However, an important drawback of the effective static stability is that it only applies to eddy motions and not the mean flow, making it difficult to form a useful set of TEM equations with the effective EP flux. Since the effective EP flux is written in terms of the effective static stability, the transformation of the zonal-mean momentum equation to include the effective EP flux divergence would require introducing a transformed streamfunction involving the effective static stability. But the zonal-mean thermodynamic equation involves a dry static stability term and a mean latent heating term, and writing it in terms of this transformed streamfunction would not eliminate the mean latent heating term.

\section{b. Moist EP flux: Equivalent potential temperature}

An alternate way to include the effects of moisture in the EP fluxes is to include moisture conservation in the governing equations and rewrite the thermodynamic equation in terms of a quantity that is conserved for moist adiabatic motions, such as equivalent potential temperature or moist static energy. This approach was introduced by Stone and Salustri (1984) to add largescale eddy forcing of condensation heating to the QG EP fluxes. Stone and Salustri (1984) did not explicitly formulate their results in terms of equivalent potential temperature ( $\theta$ in their paper is not potential temperature), but they noted that their moist thermodynamic equation expressed the conservation of equivalent potential temperature. They were then able to formulate a set of moist TEM equations and to construct a form of the nonacceleration theorem for a moist atmosphere. In our implementation of this approach, the moist EP flux is obtained by replacing $\theta$ with the equivalent potential temperature $\theta_{e}$ in Eq. (1). This is very similar to the generalized EP flux of Stone and Salustri (1984), although we allow the moist static stability in the denominator of the upward component of the EP flux to vary with latitude. It is also equivalent to the approach of Chen (2013) and Yamada and Pauluis (2016) in the QG, small-amplitude limit with a moist static stability that has a well-behaved inverse.

One drawback to this approach is that it will break down where the moist static stability $-\partial \overline{\theta_{e}} / \partial p$ is small or changes sign, and this is particularly problematic at lower latitudes. Recently this issue has been addressed by using the mass above isentropes as a vertical coordinate (Chen 2013), or a weak formulation of the mass-weighted zonal average that involves a vertical integral (Yamada and Pauluis 2016). Here, we do not want to combine contributions from the lower and middle or upper troposphere, and, since we are primarily concerned with the midlatitudes, it is sufficient for our purposes to mask out the moist EP fluxes at levels at which they are not well defined.

As pointed out by Stone and Salustri (1984), a more important drawback to this approach is that reformulating the thermodynamic equation in terms of equivalent potential temperature instead of potential temperature means that derivations in the QG framework that combine the thermodynamic equation and the hydrostatic or thermal wind equations will no longer hold. The hydrostatic and thermal wind equations involve potential temperature (or virtual potential temperature), while the thermodynamic equation now involves the equivalent potential temperature. Because of this incompatibility, it is not possible to derive a $\mathrm{QG} P V$ involving equivalent potential temperature that will be conserved for moist adiabatic and frictionless flow, and there is a similar problem for an Ertel PV defined in terms of equivalent potential temperature (Schubert 2004). As a result, it is difficult to relate the moist EP flux divergence to an eddy PV flux or to view the moist EP flux as a flux of a wave activity, at least without further approximations. Future work could investigate the utility of an approximately conserved moist PV. By contrast, using the effective static stability approach for the moist EP flux does retain the connection to eddy PV fluxes and wave activity, but it does not allow for a straightforward TEM formulation without a mean latent heating term. Therefore, the two 
moist formulations of the EP fluxes have different potential advantages and disadvantages.

\section{Dry and moist EP fluxes in a reanalysis and a GCM}

a. Climatology and projected changes

\section{1) REANALYsis: ERA-INTERIM}

We compare the moist EP flux formulations to the dry formulation using ERA-Interim data Dee et al. (2011) from 1980-2013 at a time resolution of $6 \mathrm{~h}$ and a spatial resolution of $2.5^{\circ}$ by $2.5^{\circ}$ on 37 pressure levels. All eddy quantities are calculated with respect to the zonal mean and then averaged over the time period of interest. Reanalysis output provided at pressure levels below the surface pressure is not included in the analysis. For the effective formulation of EP fluxes, the asymmetry parameter $\lambda$ is calculated at each latitude and pressure level with respect to the zonal mean and subsequently averaged in time. For the $\theta_{e}$ formulation, we calculate $\theta_{e}$ following the method of Bolton (1980). When calculating the latitude of peak surface westerlies, we take a zonal and time average of the $10-\mathrm{m}$ zonal wind data that is provided at monthly time resolution.

The dry EP flux vectors and the flux divergence $\nabla \cdot \mathbf{F}$ are shown averaged over DJF in Fig. 1a and over JJA in Fig. 1d. The EP flux is strongest in the extratropical lower troposphere of the winter hemisphere. In the midlatitudes, transient eddies generated by baroclinic processes and stationary eddies propagate vertically and are absorbed and deflected horizontally in the upper troposphere (Edmon Jr. et al. 1980; Held and Hoskins 1985; Thorncroft et al. 1993; Ait-Chaalal and Schneider 2015). Wave absorption, as indicated by the EP flux convergence, peaks around $400 \mathrm{hPa}$ in each hemisphere. Below the EP fluxes, we plot the zonal and time mean of $u \cos \phi$ at $10 \mathrm{~m}$ above the surface. We plot $u \cos \phi$ rather than $u$ because the EP flux divergence is a torque on the zonal-mean angular momentum [Eq. (3)], but for convenience we will refer to $u \cos \phi$ as the surface zonal wind or westerlies throughout this paper. The peak upward EP flux at all levels tends to be poleward of the peak surface westerlies.

Compared to the dry formulation, the EP flux in the moist formulations is stronger (Fig. 1). The effective moist formulation has a peak EP flux approximately 1.5 times stronger than that of the dry case, while the $\theta_{e}$ formulation is roughly 2.5 times stronger (note the reference vector magnitudes in each panel). These effects are strongest in the lower troposphere, and the moist formulations converge to the dry case in the stratosphere where there is little water vapor. Maximum EP flux convergence is also enhanced in the moist cases by a factor of around 1.4 for the effective formulation and 1.7 for the $\theta_{e}$ formulation.

The pattern of EP flux convergence is similar between the dry and effective moist formulations but differs in the $\theta_{e}$ formulation. Figures $1 \mathrm{c}$ and $1 \mathrm{f}$ show very large EP flux convergence in the lower troposphere in the $\theta_{e}$ formulation, and there is much less widespread EP flux divergence than in the other formulations. In the $\theta_{e}$ formulation and the effective formulation to a lesser degree, parts of the lower troposphere are masked out, especially in the tropics, where $-\partial \theta_{e} / \partial p$ and $-\partial \theta_{\text {eff }} / \partial p$ are nearly zero (or even negative), leading to unreasonably large values of upward EP flux.

One particular feature of interest in Fig. 1 is an anomalous region of EP flux divergence located at approximately $40^{\circ}$ and $250 \mathrm{hPa}$ in each winter hemisphere. This divergence feature is located where the background meridional QG PV gradient is positive (not shown), indicating upgradient eddy PV fluxes at that location, consistent with previous studies (Edmon Jr. et al. 1980; Karoly 1982; Bartels et al. 1998; Birner et al. 2013). This anomalous divergence feature is only $5 \%-10 \%$ smaller in magnitude in the moist formulations compared to the dry formulation, which suggests that moist processes are not the primary cause of this feature in ERA-Interim. However, there are a number of other regions of dry EP flux divergence in the upper and middle troposphere, particularly in the summer hemisphere, and these generally become less positive or are removed when the $\theta_{e}$ formulation of the moist EP flux is used, which suggests they may be related to moist processes.

The two moist formulations of the EP flux primarily modify the vertical component of the flux. [In the QG approximation only the vertical term would be changed, but there is also a small modification to the horizontal term in the full EP flux given by Eq. (1).] Figure 2 demonstrates the effects of moisture on $F^{(p)}$ at $700 \mathrm{hPa}$ for ERA-Interim in the annual mean. Relative to the dry case, the effective upward EP flux is stronger and peaks approximately $2^{\circ}$ of latitude farther equatorward, while the $\theta_{e}$ upward EP flux is even stronger and peaks $7^{\circ}$ and $5^{\circ}$ farther equatorward in the $\mathrm{NH}$ and $\mathrm{SH}$, respectively. While the peak surface westerlies are equatorward of the peak upward EP flux in the dry formulation, they are nearly collocated with the peak upward EP flux in the $\theta_{e}$ formulation.

\section{2) Comprehensive GCM simulations: GFDL CM3}

We next study the dry and moist formulations of the EP flux in simulations with a fully coupled atmosphere-ocean 

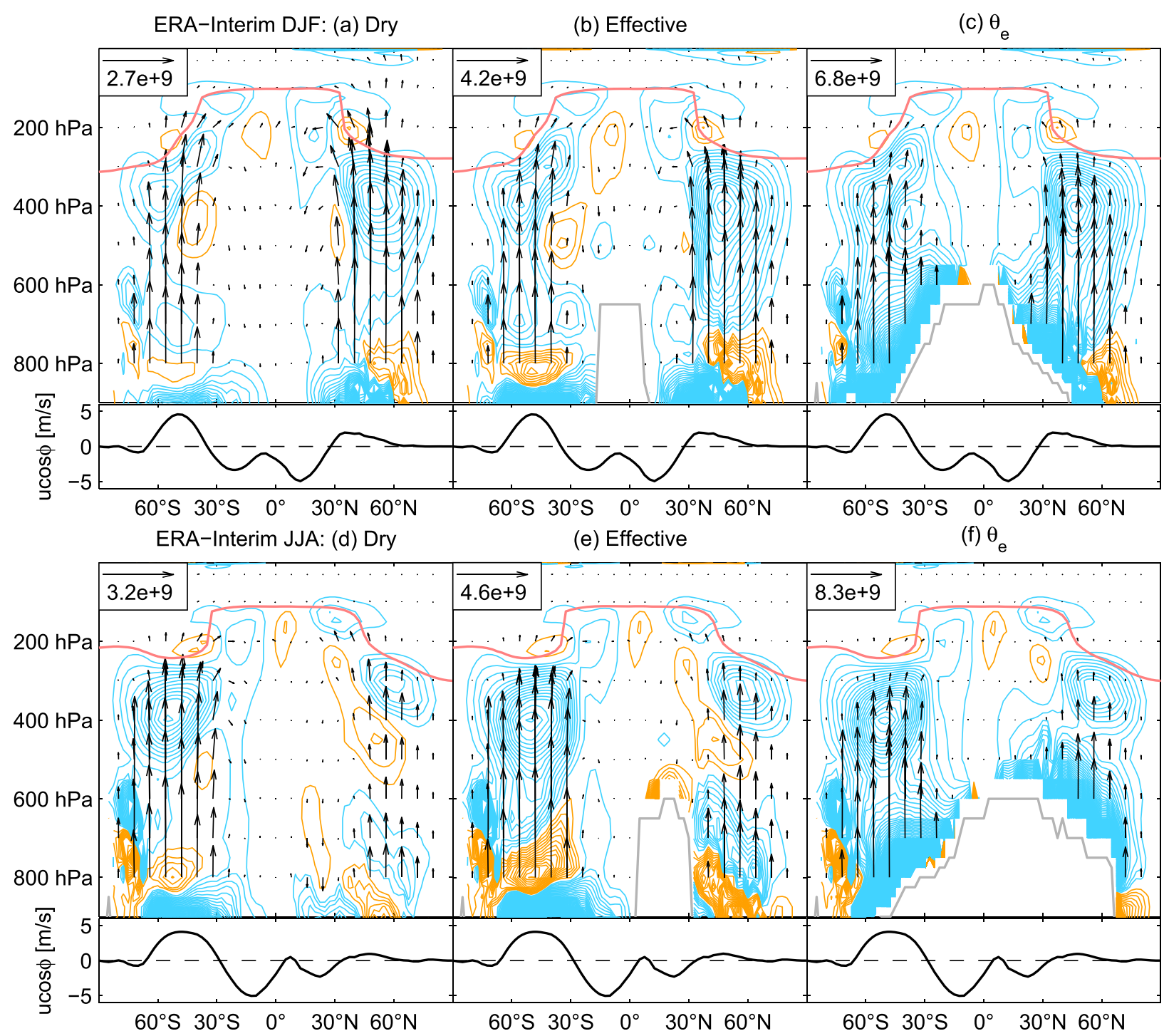

FIG. 1. The time-mean EP flux (arrows) and EP flux divergence (contours) for ERA-Interim from 1980-2013 in (top) DJF and (bottom) JJA, calculated (a),(d) with the dry theory; (b),(e) with the effective static stability; and (c),(f) with equivalent potential temperature. Contour intervals are $75 \mathrm{~m}^{2} \mathrm{~s}^{-2}$ in all panels, with blue contours indicating convergence and orange contours divergence. The horizontal arrow scale varies between panels and is given in each panel $\left(\mathrm{m}^{3} \mathrm{~s}^{-2}\right)$. A vertical arrow of the same length has a flux equal to the horizontal arrow scale multiplied by $4.5 \times 10^{-3} \mathrm{~Pa} \mathrm{~m}^{-1}$. Data are not plotted below the level at which the appropriate static stability $[-\partial \theta / \partial p$, $-(\partial \theta / \partial p)_{\text {eff }}$, or $\left.-\partial \theta_{e} / \partial p\right]$ is smaller than $0.01 \mathrm{~K} \mathrm{hPa}^{-1}$, as indicated by the gray line. The red line indicates the mean level of the tropopause using the WMO's lapse-rate definition, and the black line plot below each panel shows the zonal- and time-mean surface zonal wind as measured by $u \cos \phi$ at $10 \mathrm{~m}$ above the surface.

GCM, GFDL CM3 (Donner et al. 2011), which was included in phase 5 of the Coupled Model Intercomparison Project (Taylor et al. 2012). The GCM has a horizontal grid spacing of $2.5^{\circ}$ by $2^{\circ}$, and 6-hourly output was available on 48 model (hybrid sigmapressure) levels, spaced at roughly $50 \mathrm{hPa}$ in the upper troposphere. Vertical velocities are necessary for computing the eddy terms in the full (non QG) EP flux and for calculating the asymmetry parameter in the effective static stability. We calculated the vertical velocities from other fields following Eq. (2.5) of Simmons and Burridge (1981), and we linearly interpolated all output to pressure levels. We focus on the last two decades of the twentieth century in the historical simulation and of the twenty-first century in the RCP8.5 scenario.

Figure 3a shows the DJF climatology of the dry EP flux in GFDL CM3 averaged over the period 1980-99. Compared to ERA-Interim in Fig. 1a, the magnitude of the upward EP flux is stronger and peaks slightly equatorward. The surface westerlies in GFDL CM3 also peak 


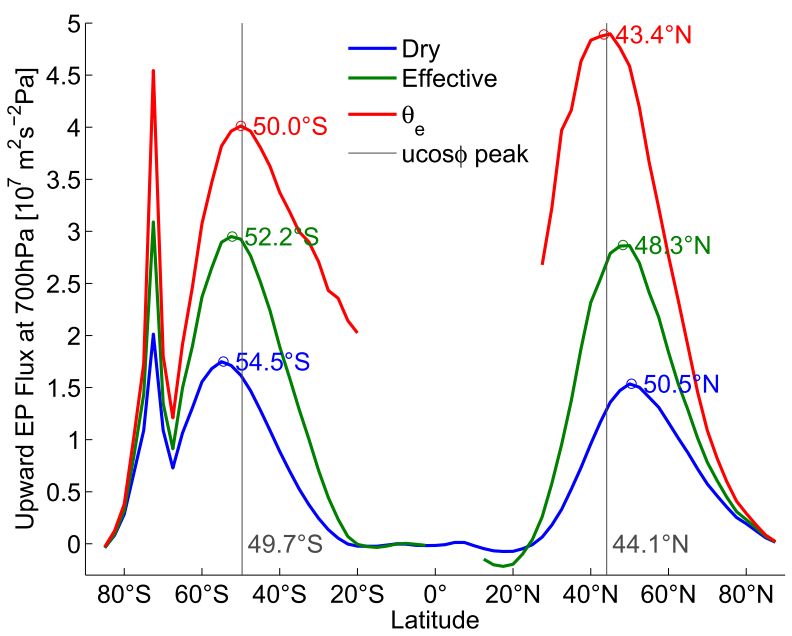

FIG. 2. The annual-mean upward $\mathrm{EP}$ flux at $700 \mathrm{hPa}$ for ERA-Interim. The dry formulation is in blue, the effective moist formulation is in green, and the $\theta_{e}$ moist formulation is in red. The gray vertical lines represent the latitude of peak surface $u \cos \phi$ in each hemisphere. The green and red curves are only plotted where their respective static stability parameters, $-(\partial \theta / \partial p)_{\mathrm{eff}}$ and $-\partial \theta_{e} / \partial p$, are larger than $0.01 \mathrm{~K} \mathrm{hPa}^{-1}$. The latitude of the peak upward $\mathrm{EP}$ flux is given in each hemisphere for each formulation. In the $\mathrm{SH}$, the peak was calculated for latitudes equatorward of $70^{\circ} \mathrm{S}$ to avoid the peak associated with Antarctic topography.

slightly equatorward compared to those in ERA-Interim, and this is a common bias in GCMs (Kidston and Gerber 2010). The EP flux convergence is also stronger in GFDL CM3 than in ERA-Interim, and this is mainly a consequence of stronger upward EP flux in the midtroposphere, since the flux is small in the upper troposphere in both cases. The level of the maximum convergence is roughly $50-100 \mathrm{hPa}$ lower in GFDL CM3 than in ERA-Interim, consistent with the lower tropopause in the former. Additionally, the anomalous region of EP flux divergence near the subtropical jet is weaker in GFDL CM3 than in ERA-Interim. The effective and $\theta_{e}$ moist EP fluxes are similarly biased when compared to ERA-Interim. As in ERA-Interim, the anomalous region of EP flux divergence near the subtropical jet persists in the moist formulations. The EP flux climatology in JJA exhibits similar biases to those in DJF (not shown). Overall, despite these biases, the main features of the EP flux climatologies are quite similar in ERA-Interim and GFDL CM3, giving confidence that the latter is adequately representing wave-mean flow interaction in the present climate.

Projected changes for the end of the twenty-first century (2080-99 minus 1980-99) in DJF in GFDL CM3 are toward overall weaker EP flux magnitudes and flux convergences in the dry case (Fig. 3d). In the winter hemisphere in the midtroposphere, the upward EP flux decreases equatorward of roughly $50^{\circ}$ and increases poleward of roughly $50^{\circ}$, indicating a poleward shift of peak upward EP flux. The dipole of decreased convergence around $400 \mathrm{hPa}$ and increased convergence around $300 \mathrm{hPa}$ is consistent with the upward shift of the tropopause and of the circulation in the middle and upper troposphere more generally (cf. Singh and O'Gorman 2012). The magnitude of the anomalous EP flux divergence feature increases in both hemispheres, but by a smaller amount than the $75 \mathrm{~m}^{2} \mathrm{~s}^{-2}$ contour interval in Fig. 3d. Changes in the moist effective case (Fig. 3e) are similar to those of the dry case in many aspects, but the decrease in midtropospheric convergence is no longer much larger in magnitude than the increase in convergence just below the tropopause in the winter hemisphere. For the $\theta_{e}$ formulation in Fig. 3f, the changes are quite different compared to the dry case. There is strengthening (rather than weakening) of the upward EP fluxes throughout the extratropical winter hemisphere and increased low-level EP flux convergence in the winter hemisphere and decreased low-level EP flux convergence in the summer hemisphere. Similar results hold for JJA, except for the $\theta_{e}$ formulation, where the changes resemble those of the effective case in the winter hemisphere, and there is strengthened low-level EP flux convergence in the summer hemisphere (not shown).

\section{b. Relationship between positions of upward EP fluxes and surface westerlies}

In an atmospheric column in the midlatitudes, the zonal-mean steady-state momentum balance is between drag on the surface westerlies and eddy momentum fluxes into the column associated with net propagation of Rossby waves meridionally out of the column. This means that the surface westerlies are not affected by the vertical position of wave activity generation within the column, but only that there is net propagation out of the column. To the extent that the waves are generated at low levels and meridional propagation occurs in the upper troposphere, there will be a link between the latitudes of the surface westerlies and the upward EP fluxes in the lower troposphere. ${ }^{1}$ Such a relationship has previously been argued for and studied in $\mathrm{Lu}$ et al. (2010) and Donohoe et al. (2014).

The prototypical life cycle of baroclinic waves is that transient eddies are generated via baroclinic instability in a relatively narrow meridional region in the lower

\footnotetext{
${ }^{1}$ An alternative approach would be to use the TEM zonal momentum balance locally to link the drag on the surface westerlies directly to the EP flux divergence near the surface, but the Coriolis torque on the residual circulation must also be taken into account, and there can be strong compensation between these terms in the lower troposphere (Pfeffer 1992).
} 

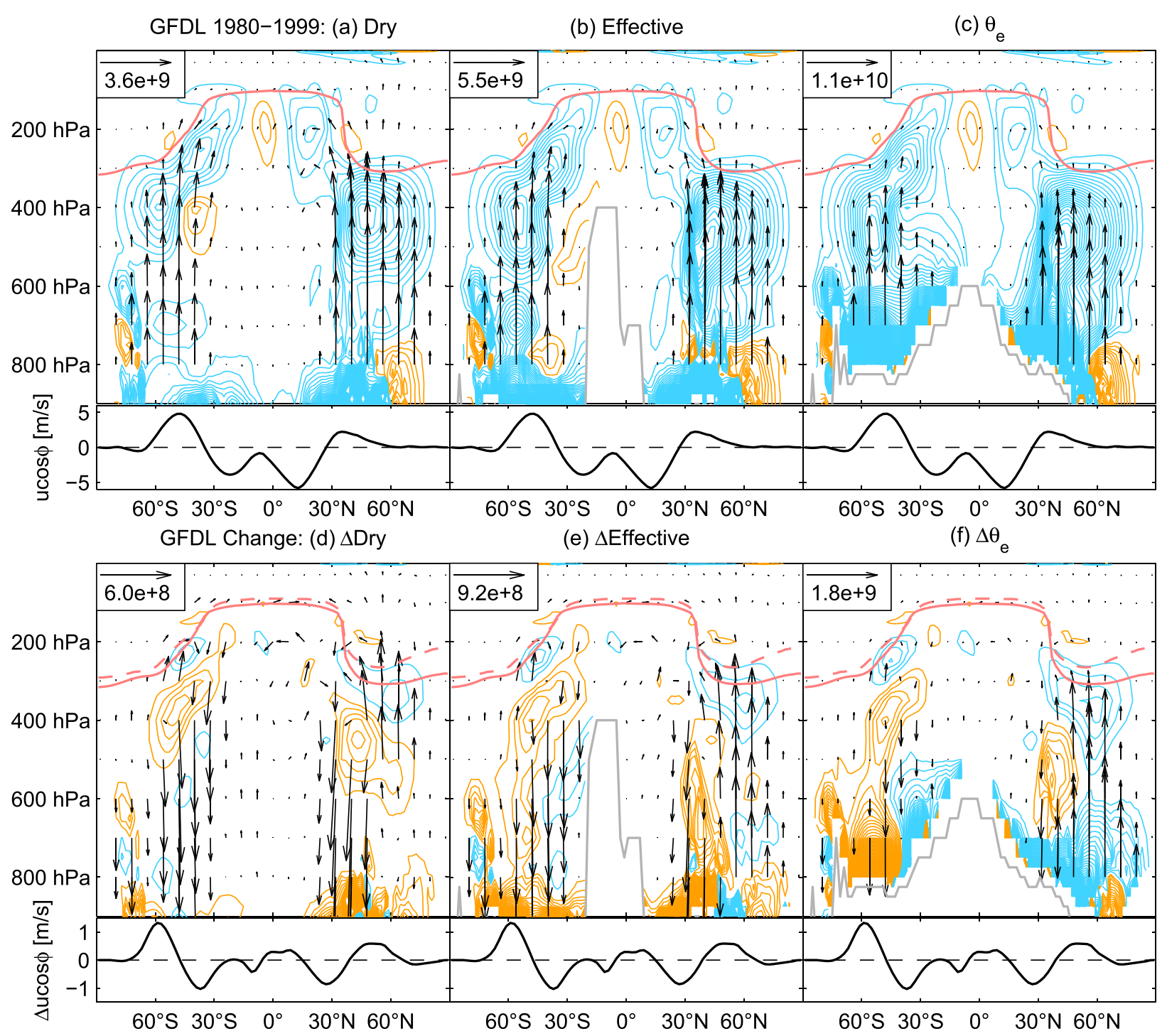

FIG. 3. As in Fig. 1, but for (top) the GFDL CM3 climatology over 1980-99 in DJF, and (bottom) the climate change for DJF defined as 2080-99 minus 1980-99 under the RCP8.5 scenario. In (bottom), the dashed red line shows the mean level of the tropopause for $2080-99$.

troposphere, propagate upward to near the tropopause, and turn and propagate meridionally (preferentially equatorward). Stationary (and quasi-stationary waves) also make a substantial contribution to the zonal momentum budget (e.g., Simpson et al. 2014), particularly in the Northern Hemisphere winter, where these waves are generated at low levels and propagate upward and then meridionally in the upper troposphere (Edmon Jr. et al. 1980). The total (transient plus stationary) EP flux shows very little meridional propagation in the lower troposphere (Fig. 1), which, as mentioned above, implies that there should be a link between the latitude of the peak upward EP flux in the lower troposphere and the latitude of the peak surface westerlies.
As we shall see in section 4, there are some striking situations in which the above argument can break down. From a dry dynamical perspective, latent heating can lead to a diabatic wave activity source in the middle or upper troposphere, and this can reduce the importance of the upward dry EP flux and break its link with the surface westerlies. More generally, to the extent that the waves are affected by moisture, we expect that using a moist version of the EP flux will give a tighter relationship between the peak upward EP fluxes and peak surface westerlies. In addition, the region of strong upward propagation has finite meridional extent (Fig. 2), and major changes in the preference for poleward or equatorward wave propagation at upper levels can move the 


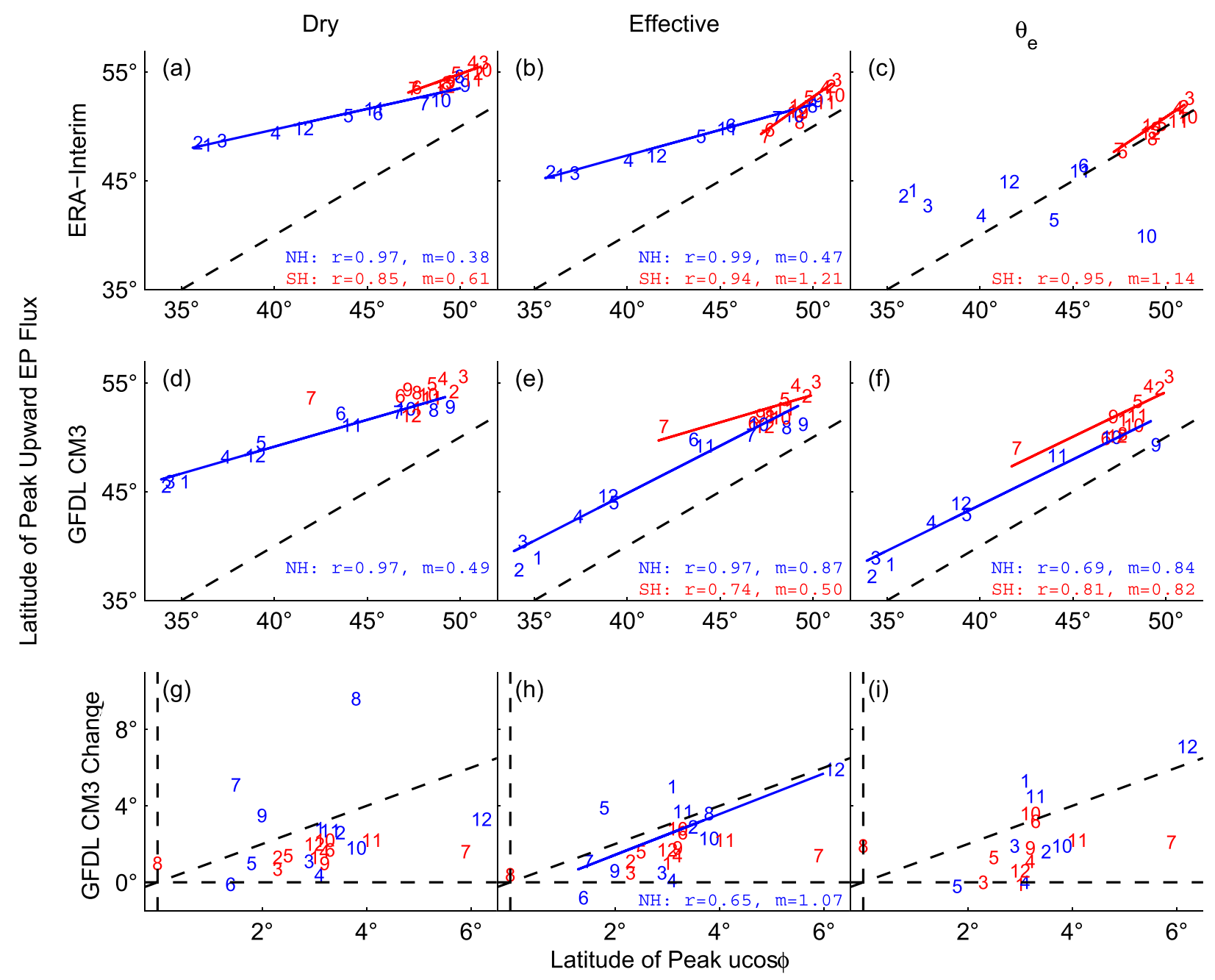

FIG. 4. Latitudes of peak upward EP flux at $700 \mathrm{hPa}$ plotted against peak surface westerlies ( $u \cos \phi)$ for (a)-(c) ERA-Interim over 19802013; (d)-(f) GFDL CM3 over 1980-99; and (g)-(i) projected changes in GFDL CM3 under the RCP8.5 scenario. Latitudes are shown over the seasonal cycle in the NH (blue) and SH (red), and the EP fluxes are evaluated using (a),(d),(g) the dry; (b),(e),(h) effective; and (c),(f),(i) $\theta_{e}$ formulations. Numbers represent the month (1 for January, 2 for February, etc.). Least squares regression lines are plotted, and the corresponding slopes $m$ and correlation coefficients $r$ are given in each panel, except where a null hypothesis of zero slope and independent monthly values cannot be rejected at the $5 \%$ level (a two-sided $t$ test is used). In the $\theta_{e}$ panels, the latitudes of peak upward EP flux for some months could not be found because of near-zero static stabilities. The dashed lines are the one-to-one and zero lines.

position of the peak eddy-momentum flux convergence (and thus the surface westerlies) within the latitude range of strong upward EP fluxes. Indeed, recent studies have emphasized the importance of changes in the index of refraction (Lorenz 2014) or in the pattern of irreversible PV mixing (Nie et al. 2016) for jet shifts, and these changes may not always lead to a simple meridional shift of the EP flux pattern.

We begin by checking whether the relationship between the upward EP flux and surface westerlies holds over the seasonal cycle. The seasonal cycle is a useful test case because the peak westerlies vary over a wide range of latitudes throughout the year. However, the seasonal cycle is a dominant signal in the atmosphere, and spurious correlations between unrelated variables may arise. Therefore, we focus on comparing the relative strengths of the relationships of the dry and moist EP fluxes to the surface westerlies.

We first study the link in ERA-Interim by plotting the latitude of peak upward dry EP flux at $700 \mathrm{hPa}$ against the latitude of peak surface westerlies as measured by $u \cos \phi$ in Fig. 4a. We calculate the latitude of the peak in each case by linearly interpolating the meridional derivative (estimated by a centered difference) in the region around the local gridpoint maximum and then finding the latitude where the derivative is zero. We only 
look for maxima equatorward of $70^{\circ} \mathrm{S}$ to avoid the peak in upward EP flux over Antarctica (see Fig. 2).

The peak latitudes of the dry upward EP flux and the surface westerlies are highly correlated with correlation coefficients of $r=0.97$ and $r=0.85$ in the $\mathrm{NH}$ and $\mathrm{SH}$, respectively. While related, the EP fluxes peak considerably farther poleward than the surface westerlies. Moreover, the least squares regression slope is much smaller than one ( $m=0.38$ in the $\mathrm{NH}$ and $m=0.61$ in the $\mathrm{SH}$ ) because the surface westerlies vary over a larger range of latitudes over the seasonal cycle as compared with the EP fluxes. Using the moist effective EP flux tightens the relationship with the surface westerlies (Fig. 4b). The correlation coefficient in the SH increases to $r=0.94$, the poleward bias of EP fluxes is reduced, and the slopes are closer to one in both hemispheres. Surprisingly, using the $\theta_{e}$ formulation does not uniformly improve on the dry relationship for ERA-Interim. While the SH gives a larger correlation coefficient and a slope closer to one than in the dry version, the $\mathrm{NH}$ does not show a strong relationship. Further analysis reveals that this may be due to small values of $\partial \theta_{e} / \partial p$ reaching well into the midtroposphere in the $\mathrm{NH}$, especially in the summer months in which local maxima of the upward EP flux cannot be found at $700 \mathrm{hPa}$ and are not plotted in Fig. 4c. Performing the analysis at a higher level in the atmosphere improves the correlation, but the slope resembles that of the dry formulation. Results for all formulations are not very sensitive to the precise pressure level chosen for the analysis, but levels near the surface suffer from small static stabilities that prevent computation of the upward EP flux, especially for the $\theta_{e}$ formulation, and levels well above the surface reduce the differences between the dry and moist formulations.

Figures $4 \mathrm{~d}-\mathrm{f}$ show that similar relationships also hold for GFDL CM3 as for ERA-Interim, except that with the $\theta_{e}$ formulation we find a tight relationship in both hemispheres. This was not the case in the NH for ERA-Interim, and the difference may result from larger static stabilities in GFDL CM3 than in ERA-Interim. The relationship in the NH in GFDL CM3 is well correlated $(r=0.69)$ and has a slope close to one $(m=0.84)$, with EP fluxes less than $5^{\circ}$ poleward of the surface westerlies. That the slope is closer to one in the moist formulations means that changes in the latitudes of the surface westerlies and upward EP flux are more similar over the seasonal cycle, highlighting the utility of the moist EP fluxes.

Next, we look at the changes in each of these quantities that occur in response to anthropogenic climate change. Figures $4 \mathrm{~g}-\mathrm{i}$ show that both the westerlies and upward EP fluxes shift poleward with warming in nearly all months of the year in both hemispheres, and this
TABLE 1. Poleward shifts in the latitude of peak surface westerlies as measured by $u \cos \phi$ at $10 \mathrm{~m}$ and upward EP fluxes in the dry, effective, and $\theta_{e}$ formulations at $700 \mathrm{hPa}$ as projected for twenty-first-century climate change in GFDL CM3. Climate change is calculated as the difference between 2080-99 in the RCP8.5 scenario and 1980-99 in the historical simulation. The poleward shifts are first calculated for each month of the year and then annually averaged.

\begin{tabular}{lcccc}
\hline \hline & $u \cos \phi$ & $F_{\text {dry }}^{(p)}$ & $F_{\text {eff }}^{(p)}$ & $F_{\theta_{e}}^{(p)}$ \\
\hline $\mathrm{NH}$ & $2.9^{\circ}$ & $2.8^{\circ}$ & $2.4^{\circ}$ & $2.8^{\circ}$ \\
$\mathrm{SH}$ & $2.9^{\circ}$ & $1.5^{\circ}$ & $1.5^{\circ}$ & $1.6^{\circ}$ \\
\hline
\end{tabular}

holds for both the dry and moist formulations. Table 1 summarizes the annual mean of these changes for both hemispheres. Poleward shifts of the surface westerlies are similar to those of the EP fluxes in the $\mathrm{NH}\left(2.9^{\circ}\right.$ vs $2.4^{\circ}-2.8^{\circ}$ ) but nearly twice as large as those of the EP fluxes in the $\mathrm{SH}\left(2.9^{\circ}\right.$ vs $\left.1.5^{\circ}-1.6^{\circ}\right)$. Unlike for the seasonal cycle, in the annual-mean response to climate change, the dry and moist EP fluxes shift meridionally by similar amounts.

Overall, the results show a strong relationship between the peak latitudes of the surface westerlies and the upward EP flux for both the seasonal cycle and climate change and that the relationship over the seasonal cycle is tighter when the moist formulations of the EP flux are used.

\section{Idealized GCM simulations}

\section{a. Model description}

We next investigate the behavior of the moist EP fluxes in idealized simulations over a wide range of climates that have been previously described in detail in O'Gorman and Schneider (2008a,b) and Schneider et al. (2010). The aquaplanet GCM used is similar to that of Frierson et al. (2006) and is based on the dynamical core of a spectral version of the GFDL GCM with T42 resolution and 30 vertical $\sigma$ levels. It has simplified radiation and moist convection schemes (Frierson 2007) as well as a zonally symmetric, static mixed layer ocean. Averages are calculated over both hemispheres and over 300 days after a 1000-day spinup from an isothermal state for each simulation. Different climates are simulated by varying two radiative parameters. The first parameter, $\alpha$, modulates the longwave optical thickness of the atmosphere, and the second parameter, $\Delta_{s}$, controls the meridional slope of insolation. The globalmean surface air temperature $T_{g}$ varies from 260 to $316 \mathrm{~K}$ approximately logarithmically with $\alpha$ between 0.2 and 6.0 for the simulations with $\Delta_{s}=1.2$, while the equator-to-pole temperature difference varies from 28 
(a) Dry

(b) Effective

(c) $\theta_{e}$ (d) Diabatic Effective

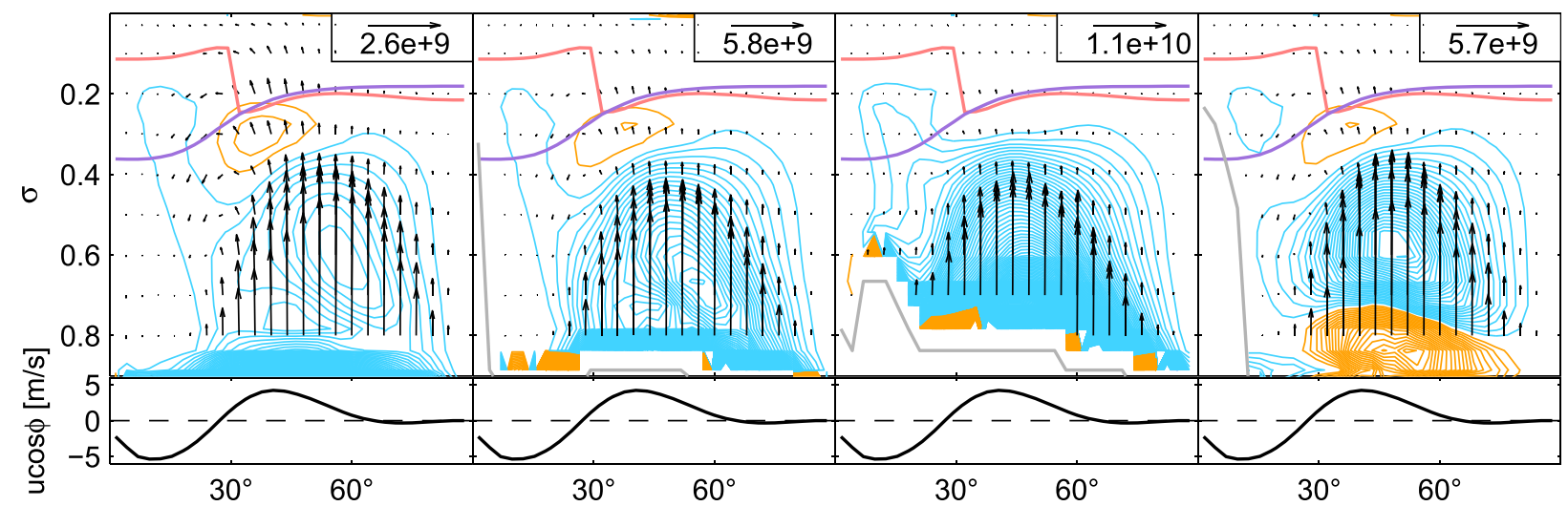

FIG. 5. As in Fig. 1, but for the idealized GCM with $\alpha=1.4$ and $\Delta_{s}=1.2$ (a relatively warm climate with a global-mean surface air temperature of $294 \mathrm{~K}$ ). The EP flux formulations are (a) dry, (b) effective, (c) $\theta_{e}$, and (d) diabatic effective, an alternate version of the effective formulation described in the text in section 4 . Vertical levels are in $\sigma$ coordinates. The purple line shows the 350-K dry isentrope for reference.

to $58 \mathrm{~K}$ roughly linearly with $\Delta_{s}$ between 0.4 and 1.6 for the simulations with $\alpha=1.0$.

The simulation with $\alpha=1.0$ and $\Delta_{s}=1.2$ has a globalmean surface air temperature and meridional temperature gradient similar to that of present-day Earth, but the idealized GCM simulations are not meant to be realistic simulations of the atmosphere because of the idealization of many physical processes and the zonally symmetric boundary conditions. In particular, the idealized GCM has no cloud radiative effects, which have been shown to be important for the poleward shift of the jet under climate change (Voigt and Shaw 2015; Ceppi and Hartmann 2016). Instead, the idealized GCM allows us to systematically investigate the dry and moist EP fluxes and their relation to the surface westerlies in a wide range of altered climate states, including very cold and dry climates and very hot and moist climates.

\section{b. EP flux climatology and anomalous EP flux divergence}

Figure 5a shows the dry EP fluxes in the idealized GCM for $\alpha=1.4$ and $\Delta_{s}=1.2$, a warm climate with $T_{g}=$ $294 \mathrm{~K}$. The idealized GCM with these parameters shows broad similarities in its EP flux climatology compared to ERA-Interim or GFDL CM3, with large-scale tropospheric convergence and preferentially equatorward EP fluxes in the upper troposphere, and an anomalous region of EP flux divergence near the subtropical jet. However, the EP flux convergence peaks considerably lower in the troposphere with no regions of EP flux divergence in the lower troposphere, and the anomalous divergence region is stronger and located lower in the troposphere.

One possible explanation for the anomalous divergence region in this model is that it is related to a source of wave activity due to moist convection or large-scale condensation (Karoly 1982). If that is the case, we would expect the anomalous region to weaken or disappear when the moist formulations of the EP flux are used. This occurs in the effective formulation in Fig. 5b with the peak EP flux divergence dropping to $152 \mathrm{~m}^{2} \mathrm{~s}^{-2}$ from the dryformulation value of $208 \mathrm{~m}^{2} \mathrm{~s}^{-2}$. The decrease in EP flux divergence is even greater in the $\theta_{e}$ formulation, with peak EP flux divergence dropping to only $59.0 \mathrm{~m}^{2} \mathrm{~s}^{-2}$ (below the smallest contour level in Fig. 5c). Other climates in the idealized model show similar reductions in the magnitude of the EP flux divergence from the dry to the $\theta_{e}$ formulation.

We also checked to see if calculating the effective EP flux using a "diabatic effective static stability" that involves fewer approximations and that accounts for all diabatic temperature tendencies (and not just latent heating) might change the results, and the resulting "diabatic effective" EP flux is plotted in Fig. 5d. ${ }^{2}$ Compared to the standard effective static stability, the diabatic effective static stability is smaller (less stable) in the middle troposphere and larger (more stable) below $850 \mathrm{hPa}$. This leads to a

\footnotetext{
${ }^{2}$ Determining the effective static stability involves calculating an asymmetry parameter $\lambda$ by regressing the eddy truncated vertical velocity (a proxy for the eddy latent heating rate) on the eddy vertical velocity, as briefly described in section $2 \mathrm{a}$. But since we store the temperature tendencies from all parameterized diabatic processes at 6-hourly resolution for the idealized GCM, we can regress the tendency of potential temperature from diabatic heating against the eddy vertical velocity directly: $Q^{\prime}=\lambda_{Q} \omega^{\prime}+\varepsilon$, where $Q^{\prime}$ is the potential temperature tendency associated with largescale condensation, moist convection, radiation, hyperdiffusion, and boundary layer diffusion, $\lambda_{Q}$ is the least squares regression slope $\left(\mathrm{K} \mathrm{Pa}^{-1}\right)$, and $\varepsilon$ is the residual. The regression is performed using the 6-hourly data at each latitude and level over all longitudes and times. We define the "diabatic effective" static stability as $-(\partial \theta / \partial p)_{\text {diabatic eff }}=-\partial \bar{\theta} / \partial p+\lambda_{Q}$, and we substitute it into Eq. (1) to give the "diabatic effective" EP flux.
} 
stronger upward EP flux and EP flux convergence in the middle troposphere and a larger EP flux divergence in the lower troposphere. But this version of the effective static stability produces an anomalous EP flux divergence near the subtropical jet that is very similar to the effective static stability formulation in Fig. 5b. We also repeated this analysis using only the moist physics tendencies rather than the full diabatic tendencies, and this resulted in negative static stabilities in the lower troposphere but did not change the anomalous EP flux divergence in the upper troposphere. Further analysis reveals a weak correlation $(r \approx-0.5)$ between the vertical velocity and the diabatic heating rate in the anomalous EP flux divergence region compared to stronger correlations in the ITCZ at $500 \mathrm{hPa}$ $(r \approx-0.9)$ or in the storm tracks at $500 \mathrm{hPa}(r \approx-0.8)$, and this is because updrafts are often not saturated in the anomalous EP flux divergence region. This weak correlation is problematic for the accuracy of the effective static stability and the diabatic effective static stability, and it may explain why the effective formulations do not weaken the divergence feature as much as the $\theta_{e}$ formulation does.

\section{c. Surface westerlies and relation to EP fluxes}

Given the strong relationship over the seasonal cycle between the surface westerlies and upward EP flux in ERA-Interim and GFDL CM3, especially for the moist EP flux formulations, we might expect a similar relationship to also hold in the idealized GCM over different climates. Remarkably, we instead find that the surface westerlies can shift equatorward with warming even when the peak upward EP fluxes (and certain other eddy-based measures such as near-surface eddy kinetic energy) shift poleward.

Figure 6 shows the latitudes of the peak surface westerlies as a function of $T_{g}$. For very cold climates in which $T_{g}<275 \mathrm{~K}$, the westerlies shift poleward with warming, except in the case of $\Delta_{s}=1.6$. For warmer climates with $275<T_{g}<295 \mathrm{~K}$, the westerlies shift equatorward with warming. The equatorward shift is accompanied by a transition in the jet structure aloft from a double jet in colder climates to a single jet in warmer climates [see Fig. 1 of Pfahl et al. (2015) for the zonal wind structure in the same simulations except at T85 resolution]. This is reminiscent of the jet transition found by Son and Lee (2005) in response to increased latent heating in a different idealized GCM. For $T_{g}>295 \mathrm{~K}$, the westerlies again tend to shift poleward or stay roughly constant in latitude with warming. By comparison, whether the storm tracks shift poleward with warming depends on how they are measured: near-surface eddy kinetic energy behaves in a similar manner to the dry EP fluxes by shifting poleward with warming (Schneider et al. 2010), whereas the latitude of peak cyclone frequency shifts equatorward with warming over part of the range of climates (Pfahl et al. 2015).
Figure 6 also shows the latitudes of the peak upward EP flux at $\sigma=0.67$. As in the analysis of GFDL CM3, this particular level was chosen as the lowest level above regions of small static stability in all formulations, but otherwise the results are not sensitive to the exact choice of level. The westerlies are generally aligned with the dry EP fluxes in cold climates. But the dry and moist EP fluxes behave differently than the surface westerlies and shift poleward to a greater extent with warming, such that in the hottest climates they are located much farther poleward than the westerlies. The moist EP fluxes are generally farther equatorward than the dry EP fluxes, and thus they are closer to the surface westerlies in the warm and hot climates.

\section{d. Dry EP flux perspective and eddy enstrophy budget}

Figure 6 also shows the latitude of the peak of the anomalous dry EP flux divergence near the tropopause and just poleward of the subtropical jet. For the hot climates, the latitude of the surface westerlies is aligned with this anomalous EP flux divergence feature. The collocation between its position and that of surface westerlies in hot climates suggests it is important for the zonal momentum budget and for the changes in the position of the surface westerlies. We will argue that the dry EP flux divergence results from a diabatic source of wave activity, and to the extent that this source results in wave activity that propagates meridional away from the source region it must affect the column zonal momentum budget and thus affect the surface westerlies. From the TEM perspective, the anomalous EP flux divergence can be linked to the surface westerlies by a contribution to the residual circulation that extends down to the surface (Haynes et al. 1991). For $\Delta_{s}=$ 1.2, the peak EP flux divergence increases with warming from roughly $10 \mathrm{~m}^{2} \mathrm{~s}^{-2}$ in the coldest climates to roughly $250 \mathrm{~m}^{2} \mathrm{~s}^{-2}$ in the simulation with $T_{g}=307 \mathrm{~K}$. In that simulation the peak EP flux convergence is only roughly $410 \mathrm{~m}^{2} \mathrm{~s}^{-2}$ (down from a peak of $1800 \mathrm{~m}^{2} \mathrm{~s}^{-2}$ in the simulation with $T_{g}=281 \mathrm{~K}$ ), which illustrates the importance of the anomalous divergence feature in warm climates.

To better understand the behavior in the anomalous dry EP flux divergence region, we analyze the eddy potential enstrophy (hereinafter eddy enstrophy) budget. ${ }^{3}$ One of the important terms in the budget is a flux-gradient term involving the meridional eddy flux of PV. By Eq. (4), a region of EP flux divergence must correspond to a northward eddy PV flux, and this PV flux is upgradient

\footnotetext{
${ }^{3}$ We find that the eddy enstrophy budget is sufficient to explain the origin of the anomalous dry EP flux divergence feature, but it would also be interesting to study this feature using a finiteamplitude wave activity budget (e.g., Nakamura and Zhu 2010) in future work.
} 


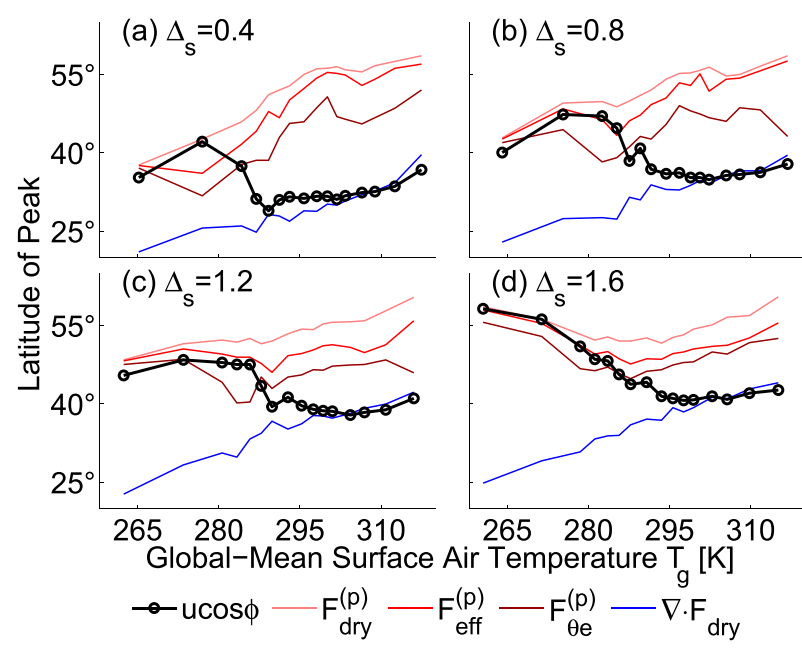

FIG. 6. Peak latitudes for various quantities as a function of global-mean surface air temperature $T_{g}$ in the idealized GCM for different values of the meridional insolation gradient parameter $\Delta_{s}$. The surface westerlies are denoted by $u \cos \phi, F_{\mathrm{dry}}^{(p)}$ is the dry upward EP flux, $F_{\text {eff }}^{(p)}$ is the upward EP flux in the effective formulation, $F_{\theta_{e}}^{(p)}$ is the upward EP flux in the $\theta_{e}$ formulation, and $\nabla \cdot \mathbf{F}_{\mathrm{dry}}$ is the anomalous region of dry EP flux divergence near the subtropical jet. The upward EP fluxes are evaluated at $\sigma=0.67$.

since the PV gradient is also northward. Studying the behavior of the other terms in the eddy enstrophy budget allows us to understand what balances the upgradient eddy PV fluxes. The eddy enstrophy budget is difficult to close, but we find that using isentropic coordinates and Ertel PV improves the budget closure. ${ }^{4} \mathrm{We}$ follow the notation of Birner et al. (2013), who applied a similar analysis for ERA-Interim, although the diabatic heating terms were not available in ERA-Interim. [See also appendix A of Jansen and Ferrari (2013) for a derivation of the adiabatic case.] The eddy enstrophy budget is given by

$$
\begin{aligned}
& \frac{1}{2} \partial_{t}{\overline{\hat{P}^{2}}}^{*}+\frac{\bar{v}^{*}}{2 a \cos \phi} \partial_{\phi}\left(\cos \phi{\overline{\hat{P}^{2}}}^{*}\right)+\frac{\bar{Q}^{*}}{2} \partial_{\theta}{\hat{P^{2}}}^{*} \\
&= \overline{\hat{S} \hat{P}}{ }^{*}-\frac{\overline{\hat{v} \hat{P}^{*}}}{a} \partial_{\phi} \bar{P}^{*}-\overline{\hat{Q} \hat{P}^{*}} \partial_{\theta} \bar{P}^{*} \\
&-\frac{1}{2 a \bar{\rho}_{\theta} \cos \phi} \partial_{\phi}\left(\cos \phi \overline{\rho_{\theta} \hat{v} \hat{P}^{2}}\right)-\frac{1}{2 \bar{\rho}_{\theta}} \partial_{\theta} \overline{\rho_{\theta} \hat{Q} \hat{P}^{2}}
\end{aligned}
$$

where $P=(\zeta+f) / \rho_{\theta}$ is the (Ertel) PV, $\rho_{\theta}=-\left(g \partial_{p} \theta\right)^{-1}$ is the isentropic density, $Q$ is the diabatic heating rate of potential temperature (i.e., the vertical velocity in

\footnotetext{
${ }^{4}$ The isentropic version of Eq. (4) [Eq. (B.15) of Andrews 1987] has additional terms, but we have confirmed that for the idealized GCM simulations the dominant balance in the divergence region is between the eddy PV flux and the EP flux divergence. This justifies our use of the eddy enstrophy budget in isentropic coordinates to study the anomalous EP flux divergence.
}

isentropic coordinates), $S$ represents sources and sinks of PV that are calculated from the parameterized tendencies of temperature and vorticity, $\bar{x}$ is now a zonal average of $x$ on an isentropic surface, $\bar{x}^{*}=\overline{\rho_{\theta} x} / \overline{\rho_{\theta}}$ is the isentropic density-weighted zonal mean, and $\hat{x}=x-\bar{x}^{*}$. In the typical steady-state picture with downgradient eddy PV fluxes, the leading-order balance is be ${ }_{\bar{*}}$ tween the first two terms on the right side of Eq. (6), and $\hat{S} \hat{P}$ is an enstrophy sink. But where the eddy PV flux is upgradient, that balance cannot hold, and other terms in the equation must play a first-order role, or $\hat{S} \hat{P} *$ must be an enstrophy source rather than a sink.

We plot each of the terms on the right-hand side of Eq. (6) in Fig. 7 for the case with $\alpha=1.4$ and $\Delta_{s}=1.2$, corresponding to $T_{g}=294 \mathrm{~K}$. [The terms on the left-hand side of Eq. (6) are all small at the levels shown.] The EP flux divergence region corresponds to negative values at around $35^{\circ}$ latitude and $350 \mathrm{~K}$ in Fig. $7 \mathrm{a}$ where the eddy PV flux has the same sign as the background PV gradient $\left(-\overline{\hat{v}}^{*} a^{-1} \partial_{\phi} \bar{P}^{*}<0\right)$. This region of upgradient eddy PV fluxes represents a sink of eddy enstrophy, and it is a much more dominant feature than the similarly located feature in ERA-interim (Birner et al. 2013). The upgradient eddy PV flux term is primarily balanced by the positive region of $-\bar{Q}_{\hat{P}}^{*} \partial_{\theta} \bar{P}^{*}$ in Fig. $7 \mathrm{~b}$. That is, the eddy enstrophy sink due to upgradient horizontal eddy PV flux is primarily balanced by the eddy enstrophy source due to downgradient vertical eddy PV flux. Note that, while the combined horizontal and vertical eddy PV flux is downgradient, it is the horizontal component that is explicitly related to the EP flux divergence.

The vertical eddy PV flux term is a diabatic source of eddy enstrophy, and it corresponds to one of the source terms in the isentropic wave activity budget of Andrews (1987). However, there are other terms in Eq. (6) that are also nonconservative (either diabatic or viscous) in nature. The term $\hat{S}^{*}$ is a sink (Fig. 7e), although it does have a positive contribution from condensational heating (not shown). The total nonconservative contribution to the eddy enstrophy budget, $-\bar{Q}^{*} \partial_{\theta}{\overline{\hat{P}^{2}}}^{*} / 2+\overline{\hat{S}}^{*}-\overline{\hat{Q}} \hat{P}^{*} \partial_{\theta} \bar{P}^{*}-{\overline{\rho_{\theta}}}^{-1} \partial_{\theta} \overline{\rho_{\theta} \hat{Q} \hat{P}^{2}} / 2$, is shown in Fig. 8a. This term largely balances $-\overline{\hat{v}}{ }^{*} a^{-1} \partial_{\phi} \bar{P}^{*}$ shown in Fig. 7a, both in the EP flux divergence region and elsewhere. There are two processes that give a positive contribution in the EP flux divergence region: heating from large-scale condensation (Fig. 8b) and longwave radiation (Fig. 8c). Of the two, the largescale condensation term's contribution is much larger in the upgradient eddy PV flux region, although the longwave radiation term does provide a smaller contribution on the flanks. The other processes contributing in Fig. 8a are moist convection, shortwave radiation, subgrid-scale boundary layer diffusion, hyperdiffusion of temperature, 


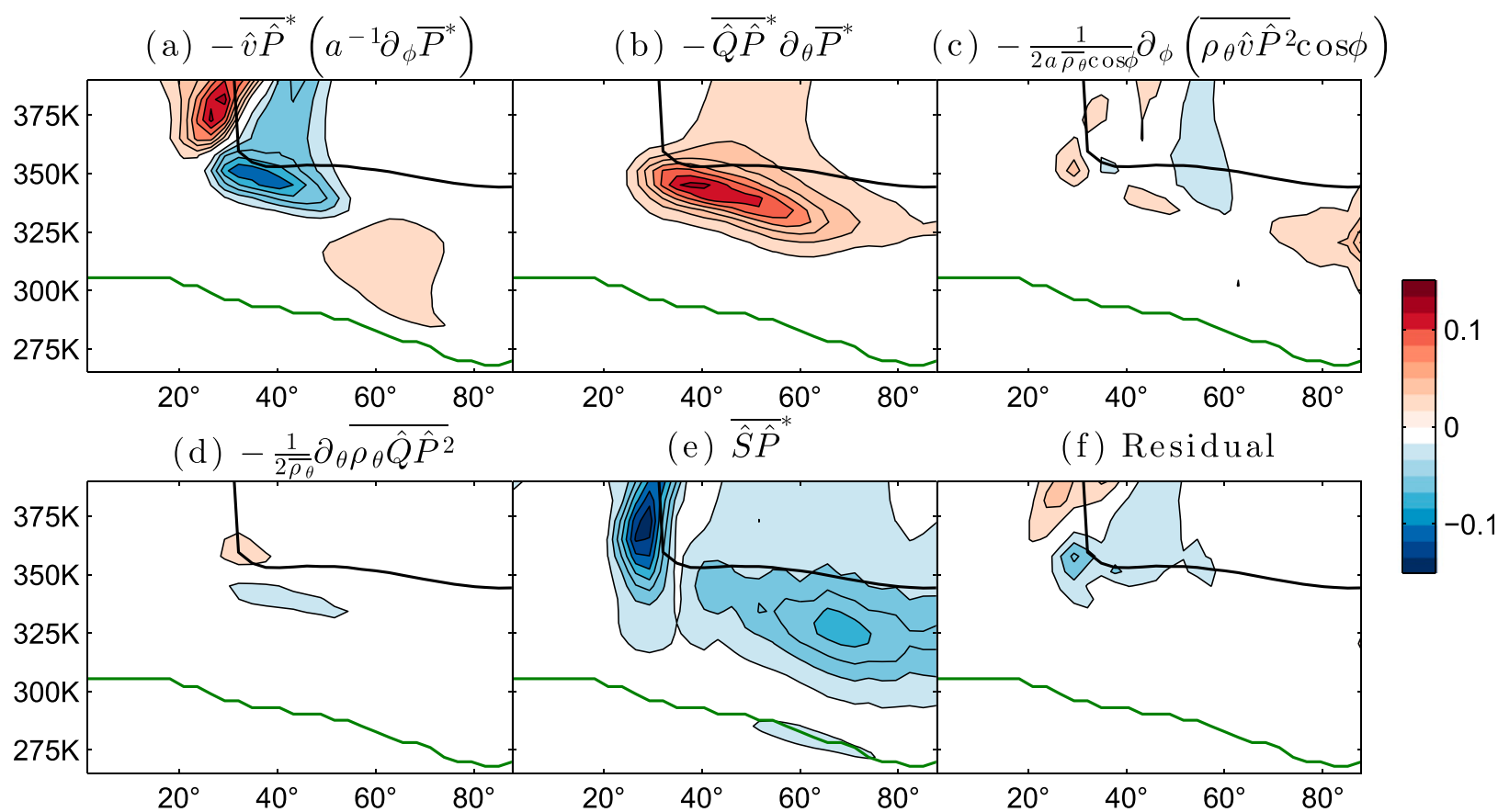

FIG. 7. The terms of the eddy enstrophy budget [Eq. (6)] for the idealized GCM with $\alpha=1.4$ and $\Delta_{s}=1.2$ in dry isentropic coordinates. All panels have contour intervals of $0.0167 \mathrm{PVU}^{2} \mathrm{day}^{-1}$. Shown are (a) the horizontal eddy PV flux term, (b) the cross-isentropic eddy PV flux term, (c) the horizontal eddy enstrophy flux convergence, (d) the vertical eddy enstrophy flux convergence, (e) the nonconservative term $\hat{S} \hat{P}$, and (f) the residual defined as the right- minus left-hand sides of Eq. (6). The black line denotes the WMO tropopause and the green line the median position of the surface. We do not plot values on levels that are below the surface $99 \%$ of the time or where $\overline{\rho_{\theta}}<10^{-6}$ $\mathrm{kg} \mathrm{K}^{-1} \mathrm{~m}^{-2}$.

and hyperviscosity. The contribution of moist convection is very small in the upgradient eddy PV flux region, which is somewhat surprising, given that $\bar{Q}^{*}$ from convection is generally much larger than $\bar{Q}^{*}$ from condensation and that $\hat{Q} \hat{P}^{*}$ of each term is comparable in peak strength (not shown). The vertical eddy PV flux term associated with condensation ends up contributing more to the eddy enstrophy source than that associated with convection because the former peaks at higher levels where the cross-isentropic PV gradient is stronger.

The other term in the eddy enstrophy budget is horizontal eddy flux convergence of enstrophy (Fig. 7c), but it is too small and not of the right spatial structure to balance $-\hat{v} \hat{P} * a^{-1} \partial_{\phi} \bar{P}^{*}$ in the upgradient eddy PV flux region. The eddy enstrophy budget does not close exactly, but the residual [the difference between the right- and left-hand sides of Eq. (6)] in Fig. $7 \mathrm{f}$ is small in the EP flux divergence region compared to the terms in Figs. 7a and 7b.

Overall, this budget analysis reveals that diabatic heating associated with large-scale condensation is responsible for the anomalous region of EP flux divergence in the idealized GCM. Thus, from the dry EP flux perspective, the relationship between the latitudes of peak surface westerlies and upward EP fluxes breaks down in warm climates in the idealized GCM because of an anomalous region of EP flux divergence, and this divergence region is associated with a diabatic source of wave activity.

\section{e. Moist EP flux perspective and changes in wave breaking}

When the moist $\theta_{e}$ formulation of the EP flux is used, the anomalous EP flux divergence greatly weakens (Fig. 5c). But although the latitude of peak upward EP flux comes closer to the latitude of peak surface westerlies in warm climates, they are still not well aligned in general (Fig. 6). The region in which the upward EP flux at $\sigma=0.67$ in the $\theta_{e}$ formulation is at least $70 \%$ of its maximum value is quite broad and covers up to $30^{\circ}$ of latitude as shown by the hatching in Fig. 9f for the $\Delta_{s}=1.2$ case. Comparing Figs. 9d and 9f, we see that peak surface westerlies are better confined to the latitude range of strong upward EP flux when the $\theta_{e}$ EP flux formulation is used as compared to the dry formulation. However, major changes in the pattern of meridional EP flux propagation in the upper troposphere move the peak eddy momentum flux convergence (and thus the surface westerlies) within this latitude range, as we next discuss.

In the cold climate in Fig. 9a, the preferred meridional direction of wave propagation in the upper troposphere is equatorward (poleward eddy momentum fluxes), and 
(a) All non-conservative

(b) Cond

(c) LW rad

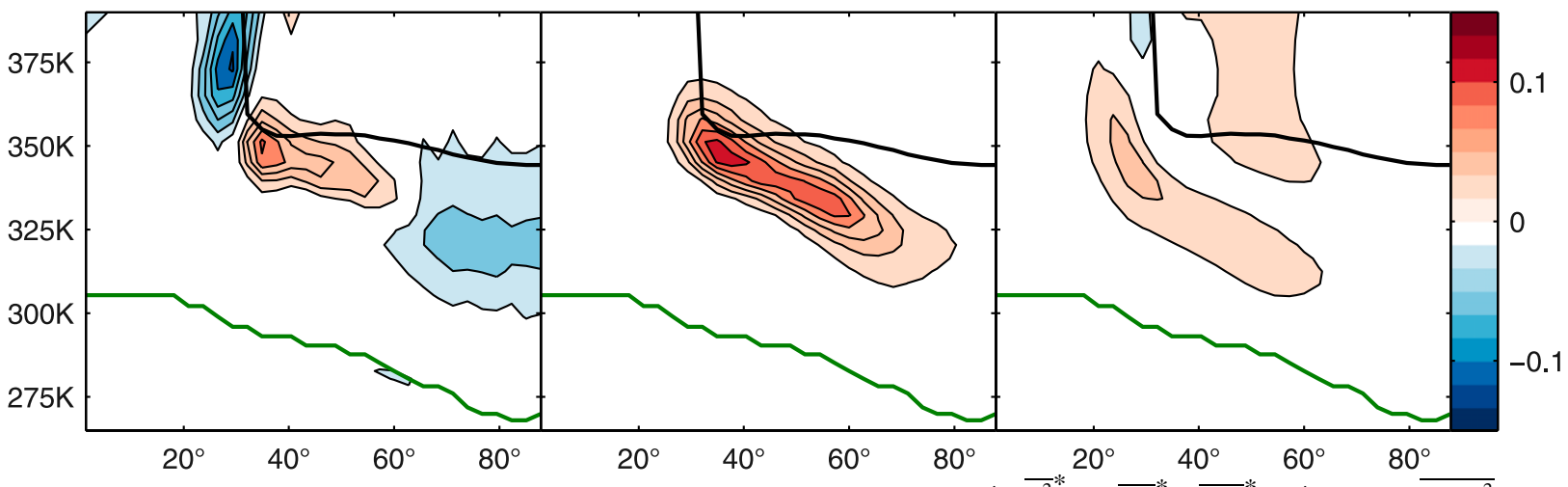

FIG. 8. The total nonconservative contribution to the eddy enstrophy budget, $-\bar{Q}^{*} \partial_{\theta}{\overline{\hat{P}^{2}}}^{*} / 2+\overline{\hat{S} \hat{P}}{ }^{*}-\overline{\hat{Q}}^{*}{ }^{*} \bar{P}_{\theta}^{*}-{\overline{\rho_{\theta}}}^{-1} \partial_{\theta}{\overline{\rho_{\theta}} \hat{Q} \hat{P}^{2}}_{2}$, for (a) all nonconservative processes, (b) large-scale condensation only, and (c) longwave radiation only. The total diabatic contribution in (a) includes the processes in (b) and (c), as well as moist convection, shortwave radiation, hyperdiffusion of temperature, boundary layer diffusion, and hyperviscosity. The contour interval and black and green lines are as in Fig. 7.

the surface westerlies peak just poleward of the latitude of peak upward EP flux in the $\theta_{e}$ formulation. ${ }^{5}$ But in warm climates like the one shown in Fig. $9 \mathrm{~b}$ and hot climates like the one shown in Fig. 9c, waves on the poleward flank of the region of strong upward EP flux propagate poleward instead of equatorward, causing the surface westerlies to peak farther equatorward in the strong upward EP flux region. Increasing poleward wave propagation is associated with an increasing frequency of cyclonic wave breaking as compared to anticyclonic wave breaking (e.g., Thorncroft et al. 1993). The greater preference for cyclonic wave breaking with warming could be related to stronger latent heat release, which increases cyclone intensity more than anticyclone intensity, leading to more cyclonic wave breaking events (Orlanski 2003; Laîné et al. 2011). Alternatively, the change in preference may relate to changes in the index of refraction associated with changes in the jet structure. Thus, to understand the changes in latitude of the surface westerlies in the idealized GCM simulations, it is necessary to take into account both the effects of latent heat release through the $\theta_{e}$ formulation of the EP flux (or through the diabatic sources of wave activity in the dry formulation) and changes in the preference for equatorward or poleward wave propagation as the climate warms.

\section{Conclusions}

In this study, we describe two formulations of moist EP fluxes, and we investigate their relationship to the surface westerlies. The effective formulation modifies

\footnotetext{
${ }^{5}$ Note that the contours in this figure show eddy momentum flux divergence, not EP flux divergence.
}

the static stability to account for latent heat release by eddies, and the $\theta_{e}$ formulation replaces potential temperature with equivalent potential temperature in the EP flux. Applying these diagnostics to ERA-Interim, we find that the magnitude of the moist EP flux vectors and EP flux convergence is much larger than in the dry version with a greater increase in the $\theta_{e}$ formulation than in the effective formulation. The enhanced EP flux convergence in the moist $\theta_{e}$ formulation of the EP fluxes must be balanced by the Coriolis force on the stronger moist residual circulation, consistent with the results of Yamada and Pauluis (2016). An anomalous region of EP flux divergence near the subtropical jet in each hemisphere is found to have a similar strength when the moist EP flux formulations are used. This suggests that the anomalous divergence is not related to moist processes, supporting the conclusions of Birner et al. (2013), who found that the EP flux divergence is related to eddy enstrophy fluxes. We also investigate the EP fluxes in the GFDL CM3 coupled GCM, and we find that in response to projected anthropogenic climate change over the twenty-first century the EP flux patterns are shifted poleward and upward. The dry and effective EP fluxes and their convergences tend to weaken, whereas there is a more mixed response for the $\theta_{e}$ formulation. We have focused on the climatology of the total EP flux, but future work could investigate the contributions to the moist EP fluxes from stationary and transient eddies and from different wavenumbers.

We find that the position of the peak upward component of the EP flux in the lower troposphere has a strong link to the position of the peak surface westerlies over the seasonal cycle in the current climate in both ERA-Interim and GFDL CM3. The slope of this relationship is much closer to one for the moist formulations of the EP flux, 
(a) $\mathrm{T}_{\mathrm{g}}=270 \mathrm{~K}$ (b) $\mathrm{T}_{\mathrm{g}}=294 \mathrm{~K}$

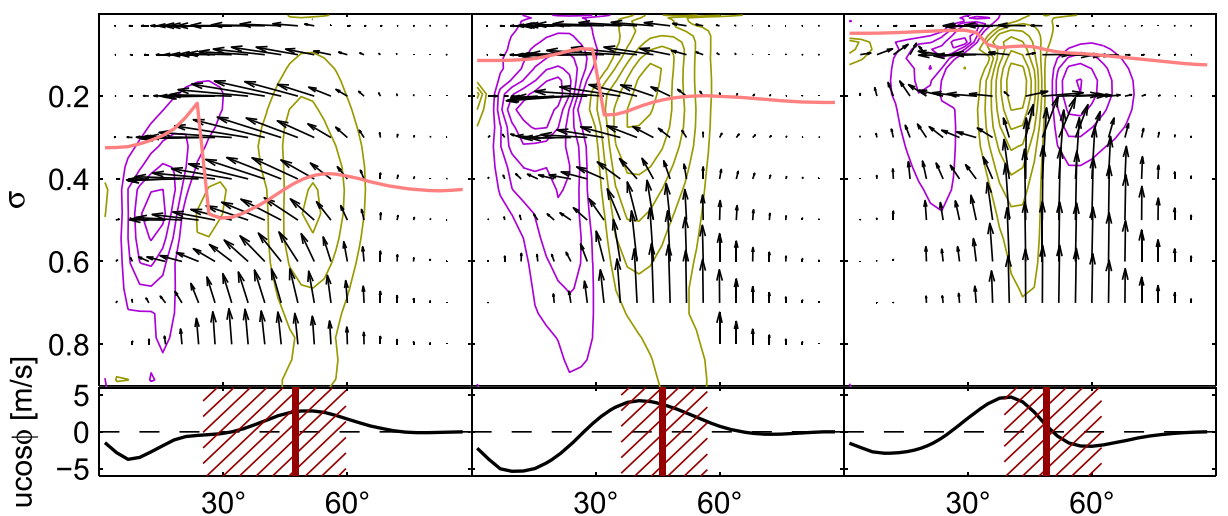

(c) $\mathrm{T}_{\mathrm{g}}=311 \mathrm{~K}$

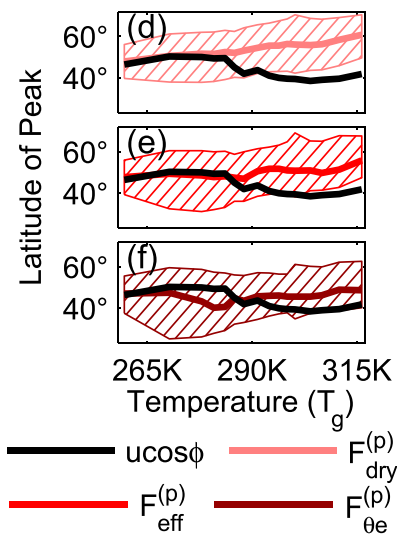

FIG. 9. (a)-(c) EP fluxes in the $\theta_{e}$ formulation and contoured horizontal eddy momentum flux divergence (not EP flux divergence) for the idealized GCM with $\Delta_{s}=1.2$ and $T_{g}$ of (a) 270, (b) 294, and (c) $311 \mathrm{~K}$. To emphasize horizontal wave propagation in the upper troposphere, the horizontal components of the EP flux vectors are first multiplied by a factor of 15 , and then the magnitudes of the vectors are multiplied by $-\log \sigma$ and then rescaled by a constant to have the same maximum length in each panel. Yellow contours indicate eddy momentum flux convergence and purple contours indicate eddy momentum flux divergence with contours every $75 \mathrm{~m}^{2} \mathrm{~s}^{-2}$. The black line plot below each panel shows the zonal- and time-mean surface zonal wind as measured by $u \cos \phi$ at $10 \mathrm{~m}$ above the surface, and solid brown lines show the latitude of peak upward EP flux in the $\theta_{e}$ formulation at $\sigma=0.67$, with hatching indicating the meridional range over which the upward EP flux is at least 70\% of its peak value. (d)-(f) As in Fig. 6c, shown are the latitude of peak surface westerlies and peak upward EP flux at $\sigma=0.67$ for $\Delta_{s}=1.2$ in the (d) dry, (e) effective, and (f) $\theta_{e}$ formulations, but also shown are the meridional range (hatching) over which the upward EP flux is at least $70 \%$ of its peak value.

and the latitude of peak upward EP flux is close to the latitude of peak surface westerlies, especially for the $\theta_{e}$ formulation. As the climate warms, both the upward EP flux and surface westerlies shift poleward in nearly all months of the year in GFDL CM3 (though there is no evidence that the poleward shifts of the upward EP flux and surface westerlies are linearly related to each other across months of the year). In the annual mean, the dry and moist EP fluxes shift poleward by the same amount, in contrast to their behavior over the climatological seasonal cycle, in which the moist EP fluxes shift over a wider range of latitudes as compared to the dry EP fluxes.

The link between the upward EP fluxes and the surface westerlies is more complicated over the wide range of climates in the idealized GCM simulations, and the surface westerlies shift equatorward with warming over part of the range of climates. This equatorward shift is reminiscent of a jet transition reported previously in response to increased latent heating (Son and Lee 2005), but here we also show that the surface westerlies can move equatorward even when the upward EP flux and certain other measures of eddy activity move poleward. From the dry EP flux perspective, the position of the surface westerlies matches that of the peak upward EP flux in cold climates, but in hot climates the surface westerlies coincide in latitude with an anomalous region of dry EP flux divergence near the subtropical jet. Investigating the eddy enstrophy budget in the idealized GCM reveals that this feature is a result of eddy enstrophy production associated with large-scale condensation, and thus the EP flux divergence may be regarded as resulting from a diabatic wave activity source. Correspondingly, the EP flux divergence in the idealized GCM is reduced when the moist versions of the EP flux are used, especially for the $\theta_{e}$ formulation, unlike for ERA-Interim. The EP flux divergence feature need not necessarily correspond to the same physical phenomenon in the idealized GCM as in ERA-Interim; the idealized GCM simulations have a different jet structure, and their EP flux divergence feature is stronger and is located somewhat lower in the atmosphere than the similar feature in ERA-Interim. On the other hand, our analysis of the idealized GCM simulations does suggest that diabatic wave activity sources have the potential to affect the surface westerlies. There are other prominent regions of dry EP flux divergence in the middle and upper troposphere in ERA-Interim, especially in the summer hemisphere, and the midtropospheric EP flux convergence in the GFDL CM3 weakens with warming. Therefore, it remains possible that interior diabatic wave activity sources could become more important for the momentum budget in warmer climates.

When the moist EP flux formulations are applied to the idealized GCM simulations, the latitude of peak upward EP flux comes closer to the latitude of peak surface westerlies in the warm and hot climates. There is also a decrease in the magnitude of the anomalous EP flux divergence (which we linked to large-scale condensation 
heating), and this feature is largely removed in the $\theta_{e} \mathrm{EP}$ flux formulation. However, even in the $\theta_{e}$ formulation there is still a discrepancy between the latitudes of the peak upward EP flux and the peak surface westerlies, which we attribute to changes in the preference for equatorward or poleward wave propagation (and anticyclonic or cyclonic wave breaking) as the climate warms. Equatorward propagation is dominant in cold climates, but waves on the poleward flank of the region of strong upward EP flux start to propagate poleward in warm climates, shifting the region of peak eddy momentum flux convergence equatorward within the latitude range of strong upward EP flux. Of course, the same changes in the meridional component of the EP flux occur when the dry EP flux is used, but when the dry perspective is used the diabatic source of wave activity must be also taken into account. The upshot is that to understand the variations in the latitude of the surface westerlies it is necessary to take account of both latent heating (either through the moist EP fluxes or as a diabatic source of dry wave activity) and changes in the preference for equatorward or poleward propagation in the upper troposphere.

Moist EP fluxes are attractive from a theoretical perspective because they take account of latent heating, which is known to affect both eddies and the mean flow. However, the moist EP fluxes discussed here do not satisfy all of the theoretical relationships that are satisfied by the dry EP flux. While the effective EP flux formulation may be connected to a wave activity and an eddy PV flux, it is difficult to form a useful set of TEM equations based on the effective static stability. The $\theta_{e}$ EP flux formulation may be connected to a TEM following the approach of Stone and Salustri (1984), and it is also related to the moist isentropic formulation of Yamada and Pauluis (2016), but it is not connected to a wave activity or eddy PV flux, and we leave to future work the question of whether this connection could be made with further approximations. Despite these theoretical drawbacks, the moist EP flux formulations do seem to have some advantages when applied to moist atmospheres since we find that they lead to a closer link between the peak latitudes of the upward EP fluxes and the surface westerlies over the seasonal cycle in reanalysis and a comprehensive GCM simulation, and the $\theta_{e}$ formulation makes it simpler to understand the behavior of the surface westerlies over a wide range of climates in the idealized GCM simulations.

Acknowledgments. We thank three anonymous reviewers for their thoughtful and helpful comments. This work was supported by NSF Grant AGS-1148594. The ERA-Interim was produced and provided by the European
Centre for Medium-Range Weather Forecasts. GFDL CM3 data are publicly available and were downloaded from the GFDL Data Portal. We thank Todd Mooring for the idea of modifying the effective static stability to directly use temperature tendencies from the physics parameterizations.

\section{REFERENCES}

Ait-Chaalal, F., and T. Schneider, 2015: Why eddy momentum fluxes are concentrated in the upper troposphere. J. Atmos. Sci., 72, 1585-1604, doi:10.1175/JAS-D-14-0243.1.

Andrews, D. G., 1987: On the interpretation of the Eliassen-Palm flux divergence. Quart. J. Roy. Meteor. Soc., 113, 323-338, doi:10.1002/qj.49711347518.

_ , and M. E. McIntyre, 1976: Planetary waves in horizontal and vertical shear: The generalized Eliassen-Palm relation and the mean zonal acceleration. J. Atmos. Sci., 33, 2031-2048, doi:10.1175/1520-0469(1976)033<2031:PWIHAV>2.0.CO;2.

_, and _- 1978: Generalized Eliassen-Palm and CharneyDrazin theorems for waves on axismmetric mean flows in compressible atmospheres. J. Atmos. Sci., 35, 509-512, doi:10.1175/1520-0469(1978)035<0509:ESPBSG > 2.0.CO;2.

—, J. D. Mahlman, and R. W. Sinclair, 1983: Eliassen-Palm diagnostics of wave-mean flow interaction in the GFDL "SKYHI" general circulation model. J. Atmos. Sci., 40, 2768-2784, doi:10.1175/1520-0469(1983)040<2768:ETWATM>2.0.CO;2.

Bartels, J., D. Peters, and G. Schmitz, 1998: Climatological Ertel's potential-vorticity flux and mean meridional circulation in the extratropical troposphere-lower stratosphere. Ann. Geophys., 16, 250-265, doi:10.1007/s00585-998-0250-3.

Birner, T., D. W. J. Thompson, and T. G. Shepherd, 2013: Up-gradient eddy fluxes of potential vorticity near the subtropical jet. Geophys. Res. Lett., 40, 5988-5993, doi:10.1002/2013GL057728.

Bolton, D., 1980: The computation of equivalent potential temperature. Mon. Wea. Rev., 108, 1046-1053, doi:10.1175/ 1520-0493(1980)108<1046:TCOEPT > 2.0.CO;2.

Ceppi, P., and D. L. Hartmann, 2016: Clouds and the atmospheric circulation response to warming. J. Climate, 29, 783-799, doi:10.1175/JCLI-D-15-0394.1.

Chen, G., 2013: The mean meridional circulation of the atmosphere using the mass above isentropes as the vertical coordinate. J. Atmos. Sci., 70, 2197-2213, doi:10.1175/JAS-D-12-0239.1.

Dee, D. P., and Coauthors, 2011: The ERA-Interim reanalysis: Configuration and performance of the data assimilation system. Quart. J. Roy. Meteor. Soc., 137, 553-597, doi:10.1002/qj.828.

Donner, L. J., and Coauthors, 2011: The dynamical core, physical parameterizations, and basic simulation characteristics of the atmospheric component AM3 of the GFDL global coupled model CM3. J. Climate, 24, 3484-3519, doi:10.1175/2011JCLI3955.1.

Donohoe, A., D. W. Frierson, and D. Battisti, 2014: The effect of ocean mixed layer depth on climate in slab ocean aquaplanet experiments. Climate Dyn., 43, 1041-1055, doi:10.1007/ s00382-013-1843-4.

Edmon, H. J., Jr., B. J. Hoskins, and M. E. McIntyre, 1980: Eliassen-Palm cross sections for the troposphere. J. Atmos. Sci., 37, 2600-2616, doi:10.1175/1520-0469(1980)037<2600: EPCSFT $>2.0 . \mathrm{CO} ; 2$.

Eliassen, A., and E. Palm, 1961: On the transfer of energy in stationary mountain waves. Geofys. Publ., 22 (3), 1-23.

Emanuel, K. A., M. Fantini, and A. J. Thorpe, 1987: Baroclinic instability in an environment of small stability to slantwise moist convection. Part I: Two-dimensional models. J. Atmos. 
Sci., 44, 1559-1573, doi:10.1175/1520-0469(1987)044<1559: BIIAEO $>2.0 . \mathrm{CO} ; 2$.

Frierson, D. M. W., 2007: The dynamics of idealized convection schemes and their effect on the zonally averaged tropical circulation. J. Atmos. Sci., 64, 1959-1976, doi:10.1175/JAS3935.1.

- I. M. Held, and P. Zurita-Gotor, 2006: A gray-radiation aquaplanet moist GCM. Part I: Static stability and eddy scale. J. Atmos. Sci., 63, 2548-2566, doi:10.1175/JAS3753.1.

Haynes, P. H., M. E. McIntyre, T. G. Shepherd, C. J. Marks, and K. P. Shine, 1991: On the "downward control" of extratropical diabatic circulations by eddy-induced mean zonal forces. J. Atmos. Sci., 48, 651-678, doi:10.1175/1520-0469(1991)048<0651: OTCOED $>2.0 . \mathrm{CO} ; 2$.

Held, I. M., and B. J. Hoskins, 1985: Large-scale eddies and the general circulation of the troposphere. Advances in Geophysics, Vol. 28, Academic Press, 3-31, doi:10.1016/S0065-2687(08)60218-6.

Jansen, M., and R. Ferrari, 2013: The vertical structure of the eddy diffusivity and the equilibration of the extratropical atmosphere. J. Atmos. Sci., 70, 1456-1469, doi:10.1175/JAS-D-12-086.1.

Karoly, D. J., 1982: Eliassen-Palm cross sections for the Northern and Southern Hemispheres. J. Atmos. Sci., 39, 178-182, doi:10.1175/1520-0469(1982)039<0178:EPCSFT >2.0.CO;2.

Kidston, J., and E. P. Gerber, 2010: Intermodel variability of the poleward shift of the austral jet stream in the CMIP3 integrations linked to biases in 20th century climatology. Geophys. Res. Lett., 37, L09708, doi:10.1029/2010GL042873.

Kushner, P. J., I. M. Held, and T. L. Delworth, 2001: Southern Hemisphere atmospheric circulation response to global warming. J. Climate, 14, 2238-2249, doi:10.1175/1520-0442(2001)014<0001: SHACRT $>2.0 . \mathrm{CO} ; 2$.

Laîné, A., G. Lapeyre, and G. Rivière, 2011: A quasigeostrophic model for moist storm tracks. J. Atmos. Sci., 68, 1306-1322, doi:10.1175/2011JAS3618.1.

Lorenz, D. J., 2014: Understanding midlatitude jet variability and change using Rossby wave chromatography: Poleward-shifted jets in response to external forcing. J. Atmos. Sci., 71, 2370 2389, doi:10.1175/JAS-D-13-0200.1.

Lu, J., G. Chen, and D. M. W. Frierson, 2010: The position of the midlatitude storm track and eddy-driven westerlies in aquaplanet AGCMs. J. Atmos. Sci., 67, 3984-4000, doi:10.1175/ 2010JAS3477.1.

Nakamura, N., and D. Zhu, 2010: Finite-amplitude wave activity and diffusive flux of potential vorticity in eddy-mean flow interaction. J. Atmos. Sci., 67, 2701-2716, doi:10.1175/2010JAS3432.1.

Nie, Y., Y. Zhang, G. Chen, and X.-Q. Yang, 2016: Delineating the barotropic and baroclinic mechanisms in the midlatitude eddy-driven jet response to lower-tropospheric thermal forcing. J. Atmos. Sci., 73, 429-448, doi:10.1175/JAS-D-15-0090.1.

O'Gorman, P. A., 2011: The effective static stability experienced by eddies in a moist atmosphere. J. Atmos. Sci., 68, 75-90, doi:10.1175/2010JAS3537.1.

— eddies in idealized simulations of changed climates. J. Climate, 21, 5797-5806, doi:10.1175/2008JCLI2099.1.

— and $-2008 \mathrm{~b}$ : The hydrological cycle over a wide range of climates simulated with an idealized GCM. J. Climate, 21, 3815-3832, doi:10.1175/2007JCLI2065.1.

Orlanski, I., 2003: Bifurcation in eddy life cycles: Implications for storm track variability. J. Atmos. Sci., 60, 993-1023, doi:10.1175/1520-0469(2003)60<993:BIELCI >2.0.CO;2.

Pauluis, O., A. Czaja, and R. Korty, 2008: The global atmospheric circulation on moist isentropes. Science, 321, 1075-1078, doi:10.1126/science.1159649.
T. Shaw, and F. Laliberté, 2011: A statistical generalization of the transformed Eulerian-mean circulation for an arbitrary vertical coordinate system. J. Atmos. Sci., 68, 1766-1783, doi:10.1175/2011JAS3711.1.

Pfahl, S., P. A. O'Gorman, and M. S. Singh, 2015: Extratropical cyclones in idealized simulations of changed climates. J. Climate, 28, 9373-9392, doi:10.1175/JCLI-D-14-00816.1.

Pfeffer, R. L., 1992: A study of eddy-induced fluctuations of the zonal-mean wind using conventional and transformed Eulerian diagnostics. J. Atmos. Sci., 49, 1036-1050, doi:10.1175/ 1520-0469(1992)049<1036:ASOEIF>2.0.CO;2.

Schneider, T., P. A. O'Gorman, and X. J. Levine, 2010: Water vapor and the dynamics of climate changes. Rev. Geophys., $\mathbf{4 8}$, RG3001, doi:10.1029/2009RG000302.

Schubert, W., 2004: A generalization of Ertel's potential vorticity to a cloudy, precipitating atmosphere. Meteor. Z., 13, 465-471, doi:10.1127/0941-2948/2004/0013-0465.

Shaw, T. A., and O. Pauluis, 2012: Tropical and subtropical meridional latent heat transports by disturbances to the zonal mean and their role in the general circulation. J. Atmos. Sci., 69, 1872-1889, doi:10.1175/JAS-D-11-0236.1.

Simmons, A. J., and D. M. Burridge, 1981: An energy and angularmomentum conserving vertical finite-difference scheme and hybrid vertical coordinates. Mon. Wea. Rev., 109, 758-766, doi:10.1175/1520-0493(1981)109<0758:AEAAMC > 2.0.CO;2.

Simpson, I. R., T. A. Shaw, and R. Seager, 2014: A diagnosis of the seasonally and longitudinally varying midlatitude circulation response to global warming. J. Atmos. Sci., 71, 2489-2515, doi:10.1175/JAS-D-13-0325.1.

Singh, M. S., and P. A. O'Gorman, 2012: Upward shift of the atmospheric general circulation under global warming: Theory and simulations. J. Climate, 25, 8259-8276, doi:10.1175/ JCLI-D-11-00699.1.

Son, S.-W., and S. Lee, 2005: The response of westerly jets to thermal driving in a primitive equation model. J. Atmos. Sci., 62, 3741-3757, doi:10.1175/JAS3571.1.

Stone, P. H., and G. Salustri, 1984: Generalization of the quasigeostrophic Eliassen-Palm flux to include eddy forcing of condensation heating. J. Atmos. Sci., 41, 3527-3536, doi:10.1175/1520-0469(1984)041<3527:GOTQGE > 2.0.CO;2.

Taylor, K. E., R. J. Stouffer, and G. A. Meehl, 2012: An overview of CMIP5 and the experiment design. Bull. Amer. Meteor. Soc. 93, 485-498, doi:10.1175/BAMS-D-11-00094.1.

Thorncroft, C. D., B. J. Hoskins, and M. E. McIntyre, 1993: Two paradigms of baroclinic-wave life-cycle behaviour. Quart. J. Roy. Meteor. Soc., 119, 17-55, doi:10.1002/qj.49711950903.

Trenberth, K. E., and D. P. Stepaniak, 2003: Covariability of components of poleward atmospheric energy transports on seasonal and interannual timescales. J. Climate, 16, 3691-3705, doi:10.1175/1520-0442(2003)016<3691:COCOPA > 2.0.CO;2.

Tung, K. K., 1986: Nongeostrophic theory of zonally averaged circulation. Part I: Formulation. J. Atmos. Sci., 43, 2600-2618, doi:10.1175/1520-0469(1986)043<2600:NTOZAC $>2.0$.CO;2.

Voigt, A., and T. A. Shaw, 2015: Circulation response to warming shaped by radiative changes of clouds and water vapour. Nat. Geosci., 8, 102-106, doi:10.1038/ngeo2345.

Yamada, R., and O. Pauluis, 2016: Momentum balance and Eliassen-Palm flux on moist isentropic surfaces. J. Atmos. Sci., 73, 1293-1314, doi:10.1175/JAS-D-15-0229.1.

Yin, J. H., 2005: A consistent poleward shift of the storm tracks in simulations of 21st century climate. Geophys. Res. Lett., 32, L18701, doi:10.1029/2005GL023684. 